\title{
Validation of a G-Band Differential Absorption Cloud Radar for Humidity Remote Sensing
}

\author{
Richard J. Roy, Matthew Lebsock, Luis Millán, And Ken B. CoOper \\ Jet Propulsion Laboratory, California Institute of Technology, Pasadena, California
}

(Manuscript received 17 July 2019, in final form 27 February 2020)

\begin{abstract}
Differential absorption radar (DAR) offers an active remote sensing solution to the problem of measuring humidity profiles with high vertical and horizontal resolution in hydrometeor layers. The Vapor In-Cloud Profiling Radar (VIPR) is a frequency-modulated continuous-wave (FMCW) G-band DAR tunable from 167 to $174.8 \mathrm{GHz}$ being developed at the Jet Propulsion Laboratory (JPL). Here we describe ground-based measurements from VIPR performed at the Department of Energy's Atmospheric Radiation Measurement (ARM) Southern Great Plains (SGP) site for humidity product validation. Two distinct measurement capabilities are investigated: 1) humidity profiles inside of cloudy volumes with $180 \mathrm{~m}$ vertical resolution, and 2) integrated water vapor (IWV) between the surface and cloud base. High radar sensitivity permits detection of upper-tropospheric clouds and retrieval of humidity profiles above $10 \mathrm{~km}$ in height. We develop an improved humidity retrieval algorithm based on a regularized least squares method that includes detailed accounting of measurement covariances and systematic error sources. This regularization mitigates high-spatial-frequency humidity biases that arise from frequency-dependent hydrometeor scattering, which is an important limitation for DAR systems. Through comparisons with over 20 coincident radiosondes, we find close agreement between in situ and remotely sensed humidity profiles, with a correlation coefficient of $r=0.96$, root-meansquare error (RMSE) of $0.8 \mathrm{~g} \mathrm{~m}^{-3}$, and median retrieval precision of $0.5 \mathrm{~g} \mathrm{~m}^{-3}$. Using a merged radiosonde and Raman lidar product for surface-to-cloud-base IWV, we demonstrate precise column sounding capabilities with $r=1.00$, RMSE of $1.2 \mathrm{~mm}$, and median retrieval precision of $0.25 \mathrm{~mm}$.
\end{abstract}

\section{Introduction}

Improving observations of vertical distributions of water vapor and temperature, often referred to as thermodynamic profiling, is a critical ingredient for furthering process-level understanding of the atmosphere (Santanello et al. 2018) and for improving numerical weather prediction forecast ability (Anderson 2018). Of particular importance for moist convective processes are the lower-tropospheric thermodynamic profiles, where spatial variability of water vapor plays a key role in dictating where convective initiation occurs and in influencing mesoscale circulation (Wulfmeyer et al. 2015). The importance of high spatiotemporal sampling of thermodynamic profiles is highlighted by the fact that passive measurements of water vapor and temperature comprise the majority of spaceborne observations assimilated in weather models. Yet, existing and proposed passive spaceborne systems are generally

Corresponding author: Richard J. Roy, richard.j.roy@jpl.nasa.gov limited to vertical resolutions-as defined in terms of the width of the so-called averaging kernel-coarser than $1 \mathrm{~km}$ in the lower troposphere (Maddy and Barnet 2008; Sahoo et al. 2015; Wulfmeyer et al. 2015), and cannot provide reliable retrievals in all measurement scenes. Microwave humidity retrievals typically include only a few vertical levels (Lipton 2003), are limited to ocean areas, and become inaccurate in the presence of precipitation (Sahoo et al. 2015). Hyperspectral infrared sounders, however, currently provide measurements of humidity profiles over land and ocean with resolution approaching $2 \mathrm{~km}$ in the lower troposphere, but are limited in coverage by the presence of optically thick clouds (Tian et al. 2019), resulting in low sampling in, for example, the cloudy tropics and midlatitude storm tracks. Ground-based passive humidity profilers have much higher vertical resolution near the surface, but this quickly degrades to $\geq 1 \mathrm{~km}$ after the lowest $\mathrm{km}$ of the atmosphere (Blumberg et al. 2015). The ability to accurately profile water vapor beneath a cloud base using ground-based passive infrared techniques has 
been demonstrated, though at the expense of information content loss relative to clear sky (Turner and Löhnert 2014).

The need for improved spaceborne observation of vertical water vapor profiles is recognized by the scientific community, with the recent decadal survey [National Academies of Sciences, Engineering, and Medicine (NASEM); NASEM 2018] naming the planetary boundary layer (PBL) as a targeted observable, and specifically calling for incubation of new space-capable technologies that can provide thermodynamic profiles near the surface with a vertical resolution of $200 \mathrm{~m}$ or better. Active remote sensing techniques are the most promising solution to this observational problem, as their vertical resolution is not fundamentally limited by broad weighting functions near the surface as in the case of passive sounders. Differential absorption lidar (DIAL) and differential absorption radar (DAR) hold particular promise for increasing spatial resolution of water vapor observations because of their precise height registration capabilities, and increasing accuracy because of the direct spectroscopic nature of the measurement (Nehrir et al. 2017). In fact, the two techniques are highly complementary because DIAL systems, such as the nascent High Altitude Lidar Observatory (HALO) being developed at NASA Langley Research Center (Nehrir et al. 2017), can measure water vapor profiles in clear air using aerosol and molecular backscatter, but cannot profile in cloudy volumes. DAR, on the other hand, relies on cloud hydrometeors in order to derive the differential absorption signal, and thus increases in precision as cloud amount increases, provided the signal is not too severely attenuated.

While DIAL is a long-established technique for both ground-based (Browell et al. 1979; Nehrir et al. 2011; Spuler et al. 2015; Weckwerth et al. 2016) and airborne (Browell et al. 1998) water vapor profiling measurements, DAR is a recently emerging humidity remote sensing technology that utilizes a wideband radar transmitter to measure cloud and precipitation reflectivity profiles at multiple frequencies near an $\mathrm{H}_{2} \mathrm{O}$ rotational absorption line. Additionally, DAR has been investigated in recent instrument simulator studies showing promise for spaceborne water vapor measurement applications (Lebsock et al. 2015; Millán et al. 2016; Battaglia and Kollias 2019). Unlike wavelengths for DIAL systems, where many atmospheric gases feature a plethora of ro-vibrational transitions (e.g., $\mathrm{H}_{2} \mathrm{O}, \mathrm{CH}_{4}$, and $\mathrm{CO}_{2}$ ), the microwave and millimeter-wave spectrum features only a handful of absorption lines from $\mathrm{O}_{2}$ and $\mathrm{H}_{2} \mathrm{O}$. While it has been proposed to utilize the water vapor line at $22 \mathrm{GHz}$ for DAR (Meneghini et al. 2005), such a system would be limited to sampling precipitating volumes. The next higher-frequency line location is $183 \mathrm{GHz}$, implying that developing such a DAR system also requires G-band radar innovation. While this requirement brings added technical complexity, it promises added benefits for developing such a system, as G-band radar opens new remote sensing opportunities for studies of cloud microphysics (Battaglia et al. 2014), especially in the context of multifrequency radar observations. Furthermore, the short radar wavelength gives G-band DARs increased sensitivity to small cloud particles, allowing for profiling in a wide variety of cloud scenes and precipitation.

In this work, we report on the validation of groundbased, G-band DAR measurements of vertical water vapor profiles inside of clouds, as well as measurements of column-integrated water vapor between the surface and cloud base. The instrument, called the Vapor InCloud Profiling Radar (VIPR), features hardware upgrades that significantly increase the radar's sensitivity relative to our previous work (Roy et al. 2018) in which the DAR technique was demonstrated, but not validated with ancillary water vapor measurements. We observe continuous cloud profiles and retrieve continuous water vapor profiles from the surface to above $10 \mathrm{~km}$ in height. Using an improved DAR inverse algorithm that incorporates systematic uncertainties into the retrieval, we present the first-ever validated, remotely sensed measurements of water vapor inside of clouds, and demonstrate strong agreement between DAR and in situ humidity measurements within the PBL and free troposphere.

\section{Instruments and measurement methods}

The validation measurements described in this work were performed during an intensive observation period on site at the ARM Southern Great Plains (SGP) central facility from 2 to 14 April 2019. The primary measurements used for DAR humidity validation are radiosonde humidity profiles, since existing humidity remote sensing systems are unreliable inside of clouds. Additionally, water vapor profiles from the ARM Raman lidar allow for high-temporal-resolution comparisons with DAR measurements of integrated water vapor (IWV) between the surface and cloud base.

\section{a. G-band DAR: VIPR}

VIPR is a proof-of-concept DAR being developed at the Jet Propulsion Laboratory (JPL) for remote sensing of water vapor in cloudy volumes, specifically targeting the PBL. In addition to being the first water vapor DAR, VIPR is the first all-solid-state G-band cloud radar, and is operated in frequency-modulated, continuous-wave 
(FMCW) mode to increase the transceiver sensitivity relative to a pulsed system. An early version of the VIPR system, discussion of FMCW radar detection, and demonstration of the DAR measurement principle were previously described in detail in Cooper et al. (2018) and Roy et al. (2018). Since this early work, a higher-power transmitter and higher-gain optics have been installed, greatly increasing the sensitivity of the system, and the hardware has been modified for aircraft deployment. Technical details of the aircraft-compatible VIPR system can be found in Cooper et al. (2020), while relevant details of the instrument hardware for this work are discussed below and summarized in Table 1. Figure 1 shows VIPR during the ARM SGP deployment in both calibration and cloud-measurement configurations.

The radar features a state-of-the-art, all-solid-state transmitter that is tunable between 167 and $174.8 \mathrm{GHz}$ with $200 \mathrm{~mW}$ of $\mathrm{CW}$ output power, leveraging significant research and development in high-power millimeterwave generation at JPL using GaAs Schottky diode frequency multiplication technology (Siles et al. 2018). The chosen radar band is a result of international regulations [National Telecommunications and Information Administration (NTIA); NTIA 2015] that prohibit G-band transmission in spectral regions reserved for passive satellite sensors, including the $174.8-191.8 \mathrm{GHz}$ band used for humidity sounding. As a result, VIPR is positioned on the low-frequency flank of the $183 \mathrm{GHz}$ water vapor absorption line, which makes the system primarily sensitive to PBL water vapor and allows the radar beam to penetrate through the whole atmosphere, whereas frequencies closer to the line center would experience too much absorption to feasibly probe all levels. As compared with high-peak-power, pulsed cloud radars with transmitter duty cycles on the order of $1 \%$, the use of FMCW detection makes VIPR's timeaveraged output power equivalent to a $20 \mathrm{~W}$ pulsed system. Furthermore, the short wavelength of $\lambda=1.8 \mathrm{~mm}$, large primary aperture gain, and low system noise figure result in a radar signal-to-noise ratio (SNR) of unity for a cloud reflectivity of $-40 \mathrm{~dB} Z$ at $1 \mathrm{~km}$ range using nominal radar acquisition parameters (see Table 1).

Data acquisition and signal processing are performed on a rack-mounted PC, after the FMCW radar signal has undergone analog pulse compression in the front-end G-band mixer and subsequent I/Q down-conversion to baseband. A single computer program controls the necessary components for radar signal generation (e.g., chirp initiation, transmit frequency switching), digitizes the complex I/Q baseband signal, performs digital signal processing in parallel with radar signal acquisition, and provides a real-time display of the FMCW baseband
TABLE 1. VIPR hardware and radar signal acquisition parameters.

\begin{tabular}{lc}
\hline \multicolumn{2}{c}{ Transmitter/receiver } \\
\hline W-band input power & $1.6 \mathrm{~W}$ \\
G-band output power & $0.2 \mathrm{~W}$ \\
System noise figure & $10 \mathrm{~dB}$ \\
Antenna diameter & $0.6 \mathrm{~m}$ \\
$3 \mathrm{~dB}$ beamwidth & $0.24^{\circ}$ \\
Antenna gain (calculated) & $58 \mathrm{~dB}$ \\
Beam polarization & Circular \\
\multicolumn{1}{c}{ Nominal radar signal acquisition parameters } \\
\hline Online frequency & $174.8 \mathrm{GHz}$ \\
Offline frequency & $167 \mathrm{GHz}$ \\
Transmitter frequency & $80 \mathrm{~Hz}$ \\
$\quad$ switching rate & \\
Reflectivity detection sensitivity & $-40 \mathrm{dBZ}$ for SNR $=1 \mathrm{at} 1 \mathrm{~km}$ \\
Time-domain window & $\mathrm{Hann}$ \\
Range sidelobe suppression & $-23 \mathrm{~dB}$ \\
Number of chirps per frequency & 2000 \\
Chirp duration & $1 \mathrm{~ms}$ \\
Chirp bandwidth & $10 \mathrm{MHz}$ \\
Range resolution & $15 \mathrm{~m}$ \\
Radar duty cycle & $80 \%$ \\
\end{tabular}

time-domain signal, resulting power spectra, and 2D plots of cloud reflectivity. Digital signal processing steps include the application of a time-domain window to a buffered set of 400 radar pulses, computation of individual power spectra using fast Fourier transforms (FFTs), and averaging of the resulting power spectra. For memory considerations, spectral averages are computed for sets of 2000 pulses at each frequency and then stored in permanent memory. For the typical acquisition parameters in Table 1, a single logged measurement for two transmit frequencies takes $5 \mathrm{~s}$, which sets the smallest time resolution for humidity profiling, and during which we rapidly switch between transmitter frequencies at a rate of $80 \mathrm{~Hz}$.

Differential absorption measurements using cloud radars feature a few important distinctions from DIAL systems. First, the large width of molecular absorption lines at millimeter and submillimeter wavelengths in the troposphere requires that the "online" and "offline" transmitter positions span a fractional bandwidth of $1 \%-10 \%$, while for DIALs this number is typically around $0.01 \%$. Second, the scattering targets of interest for DAR are the hydrometeors that make up clouds and precipitation, while for DIAL, the dominant lidar signal originates from atmospheric aerosols. Because characteristic diameters of hydrometeor drop size distributions (DSDs) can vary by large amounts over small spatial scales, these two factors make DAR susceptible to systematic measurement biases stemming from frequencydependent scattering. Furthermore, because the observed 

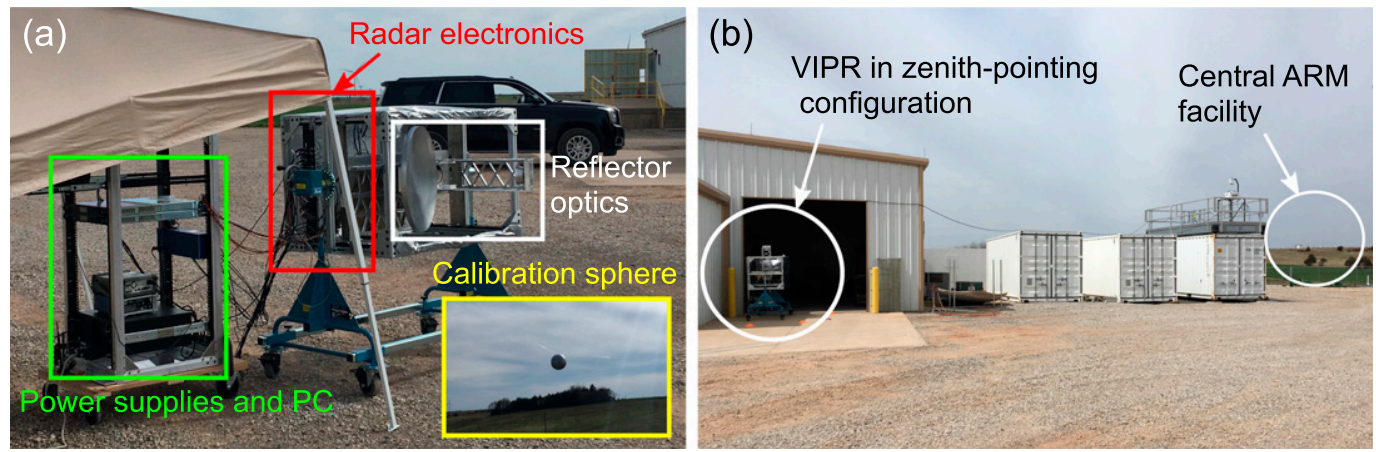

FIG. 1. VIPR deployment at the ARM SGP site in (a) calibration sphere measurement configuration and (b) zenith-pointing cloud measurement configuration. The radiosonde launches, Raman lidar, and calibration sphere are located at the central facility, with approximate distances of 900, 610, and $460 \mathrm{~m}$ from VIPR, respectively.

cloud and precipitation scenes can change quickly in time (e.g., from advection and sedimentation), it is critical to switch between DAR frequencies rapidly, as otherwise temporal dependence of reflectivity would be indistinguishable from frequency dependence and therefore humidity.

To mitigate the potential biases from frequencydependent hydrometeor scattering, we implement a new DAR retrieval method that is based on a regularized least squares approach and is discussed in section 3b. Furthermore, to limit the deleterious effects from cloud scene dynamics we switch between transmit frequencies after 10 pulses of $1 \mathrm{~ms}$ duration at each frequency. With a $2 \mathrm{~ms}$ switching time for the Ka-band synthesizers that generate the radar signal, this results in a transmitter duty cycle of $80 \%$. We note that in recent instrument simulation work, Battaglia and Kollias (2019) employed a retrieval that includes an extra fitting parameter to independently extract the spectral dependence of hydrometeor scattering and thus retrieve an unbiased humidity. However, this requires a large transmitter bandwidth to distinguish the linear frequency dependence of hydrometeor scattering from the higher-order dependence of the water vapor absorption coefficient. For VIPR, the transmit band from 167 to $174.8 \mathrm{GHz}$ features little curvature of the water vapor absorption coefficient, and is fixed by a combination of international regulations and hardware constraints. For these validation measurements, we employ a two-frequency differential absorption measurement approach, with offline frequency $f_{1}=167 \mathrm{GHz}$ and online frequency $f_{2}=174.8 \mathrm{GHz}$.

The primary quantity measured by VIPR is the radar echo power as a function of range and frequency,

$$
P_{e}(r, f)=\frac{Z(r, f) e^{-2 \tau(r, f)}}{C(f) r^{2}}=\frac{Z_{\mathrm{obs}}(r, f)}{C(f) r^{2}} .
$$

Here $Z(r, f)$ and $Z_{\text {obs }}(r, f)$ are the unattenuated and observed reflectivity factors,

$$
\tau(r, f)=\int_{0}^{r} d r^{\prime}\left[\beta_{g}\left(r^{\prime}, f\right)+\beta_{h}\left(r^{\prime}, f\right)\right]
$$

is the one-way optical depth, $\beta_{g}$ and $\beta_{h}$ are the gaseous and hydrometeor absorption coefficients, and $C(f)$ is a radar hardware calibration factor. The procedure for determining $C(f)$ and thus calibrating reflectivity profiles is outlined in the appendix. For DAR measurements of water vapor profiles inside of clouds, the value of $C(f)$ is not needed because the retrieval involves the ratio of $P_{e}(r, f)$ at two different ranges. Thus, the relevant sources of uncertainty in the retrieval of humidity are the random error in the measurement of $P_{e}$ and the systematic uncertainty related to the frequencydependent scattering parameters and stemming from our lack of knowledge about the true hydrometeor DSD. The systematic uncertainty terms will be discussed in more detail in section $3 b$.

In previous work (Roy et al. 2018), we demonstrated that the distribution of measured radar echo powers follows the well-known radar speckle model for randomly distributed scatterers, with resulting variance

$$
\operatorname{var}\left[P_{e}(r)\right]=\frac{P_{e}^{2}(r)}{N}\left(1+\frac{2}{S_{n}}+\frac{2}{S_{n}^{2}}\right),
$$

where $S_{n}=P_{e}(r) / P_{n}(r)$ is the signal-to-noise ratio, $P_{n}(r)$ is the receiver noise power in range bin $r$, and $N$ is the number of independent pulses averaged to obtain the echo power estimate. In contrast to pulsed radar systems, we write the noise power in FMCW radar explicitly as a function of range because of the mapping of range to frequency and the potential for nonuniform gain and noise power as a function of frequency in the 
baseband electronics. Additionally, the application of a time-domain window to the baseband radar signal prior to computing the power spectrum introduces covariance between neighboring range bins. The specific form of this covariance term can be derived by invoking the convolution theorem, which allows one to express the complex Fourier amplitudes of the windowed time-domain signal as a linear combination of those from the unwindowed signal, which themselves are statistically independent. The mean values and covariance matrix for the radar echo power spectrum are then computed using the squared moduli of the windowed amplitudes. For the Hann window used in this work, nearest neighbors have covariance

$$
\operatorname{cov}\left[P_{e}(r), P_{e}(r \pm \Delta r)\right]=\frac{4 P_{e, \pm}^{2}}{9}\left(1+\frac{2}{S_{n, \pm}}+\frac{2}{S_{n, \pm}^{2}}\right),
$$

where $P_{e, \pm}=\left[P_{e}(r)+P_{e}(r \pm \Delta r)\right] / 2, S_{n, \pm}=2 P_{e, \pm} /\left[P_{n}(r)+\right.$ $\left.P_{n}(r \pm \Delta r)\right]$, and $\Delta r$ is the radar range resolution. In FMCW radar, the range resolution is given by $\Delta r=$ $c / 2 \Delta F$, where $c$ is the speed of light and $\Delta F$ is the frequency chirp bandwidth. In this work, the nominal chirp bandwidth of $\Delta F=10 \mathrm{MHz}$ results in a range resolution of $\Delta r=15 \mathrm{~m}$. Therefore, the measurement covariance matrix, which we will need for the regularized least squares retrieval in section $3 b$, is tridiagonal.

\section{b. ARM instruments}

The primary measurement used for DAR humidity validation comes from the radiosondes (Vaisala RS41SG) launched from the ARM SGP central facility, located about $900 \mathrm{~m}$ NW of the radar's location. In addition to the nominal ARM radiosonde (RS) launches at 0000, 0600, 1200, and 1800 UTC, there was an increased frequency of RS launches as high as twice per hour when thick clouds were present. The typical ascent rates were between 5 and $7 \mathrm{~ms}^{-1}$, and temperature, relative humidity (RH), and GPS height were recorded every second. In this work, we report all height coordinates referenced to mean sea level. The radiosondes quickly advected away from the ARM site after launch with an average horizontal displacement of $30 \mathrm{~km}$ at $10 \mathrm{~km}$ height. While this introduces the potential for collocation errors between in situ and remotely sensed measurements, we find that humidity profiles from RS launches spaced $30 \mathrm{~min}$ apart show little variation on the scale of DAR humidity errors. Therefore, in our analysis we treat the entire profile measurement as having occurred at the time of launch. From the RH and temperature accuracy values reported on the Vaisala RS41-SG datasheet, we calculate an absolute humidity
RS accuracy of $0.7,0.4,0.2$, and $0.1 \mathrm{~g} \mathrm{~m}^{-3}$ at $20^{\circ}, 10^{\circ}, 0^{\circ}$, and $-10^{\circ} \mathrm{C}$, respectively.

In fact, for water vapor profiling inside of clouds, the RS in situ measurement is the only reliable one out of all the ARM instruments, since existing passive and active remote sensors are incapable of making this measurement accurately. However, for the measurements of IWV between the surface and cloud base, we utilize water vapor profiles retrieved using the ARM Raman lidar (RL) in addition to the RS values (Turner et al. 2016). The RL transmits a near-UV ( $355 \mathrm{~nm})$, highpeak-power pulse, and detects the inelastically scattered photons from particular $\mathrm{H}_{2} \mathrm{O}$ and $\mathrm{N}_{2}$ vibrational transitions. The range-resolved ratio of detected inelastically scattered photons from $\mathrm{H}_{2} \mathrm{O}$ and $\mathrm{N}_{2}$ provides a profile that is proportional to the water vapor mixing ratio, with a multiplicative constant provided by an ancillary sensor for absolute calibration (Whiteman et al. 1992; Turner and Goldsmith 1999). The spatial and temporal resolution of the water vapor product used here are $60 \mathrm{~m}$ and $10 \mathrm{~s}$ (ARM 2015). In between radiosonde launches the RL provides valuable independent water vapor profiles with high temporal resolution that we utilize in the IWV measurement comparisons.

\section{Retrieval methodology}

The DAR humidity measurement principle is to use the spectral variation of $Z_{\mathrm{obs}}(r, f)$ and known absorption line shape to estimate the water vapor density along the beam path. With continuous profiles of reflectivity over an extended range (e.g., from clouds and precipitation), one can use $Z_{\text {obs }}$ measurements at two ranges $r_{1}$ and $r_{1}+R$ to remove the absorption contribution between the radar and $r_{1}$ as well as the dependence on radar calibration, and thus retrieve the average absolute humidity between these two ranges. Alternatively, if there is a measurement at a single range (e.g., Earth's surface for an airborne DAR or a cloud base for a ground-based system), knowledge of the relative radar calibration for all transmitted frequencies allows for a measurement of the corresponding IWV.

In the case of the two-range measurement, varying the starting position $r_{1}$ throughout the cloud returns the full in-cloud water vapor profile, with effective resolution $R$. If the retrieval resolution is coarser than the radar range resolution, that is, if $R>\Delta r$, this approach leads to oversampling of the humidity profile. Ideally, one would set $R$ equal to the radar range resolution to maximize the humidity profile resolution. However, several constraints on DAR systems make it the case that $R$ is typically greater than $\Delta r$. First, the difference in absorption cross section at the online and offline 
frequencies and desired humidity accuracy set the achievable resolution $R$, which in the case of VIPR is constrained by the allowable transmission band. In fact, this consideration suggests the use of nonuniform vertical spacing in the retrieved humidity profile, with resolution decreasing as a function of altitude due to the smaller differential absorption cross section. However, in this work we adopt uniform vertical spacing to simplify the analysis and because we anticipate averaging the retrieved profiles in time to increase precision. Second, radar speckle noise presents a fundamental limit on the reflectivity precision within a single range bin even for infinite SNR. Thus, it is desirable to use the smallest range bin size possible and therefore acquire more independent power samples. While one could subsequently average adjacent range bins down to the resolution $R$, cloud reflectivities can easily vary by more than an order of magnitude over the scale of the achievable humidity resolution $R$, and therefore, such averaging erases information that could be utilized in the retrieval.

In this section, we present a new DAR inversion method that retrieves the humidity profile at the downsampled resolution $R$ while utilizing all reflectivity measurements at resolution $\Delta r$, resulting in no oversampling of the humidity profile. Furthermore, the least squares approach employed in this retrieval simplifies the accounting of covariance terms between adjacent ranges, and allows for the inclusion of systematic uncertainty as well as regularization to mitigate the potential biases from frequency-dependent scattering. Below we summarize the standard retrieval approach for differential absorption measurements to provide context before presenting the new algorithm.

\section{a. Standard differential absorption retrieval}

The standard DAR approach (Roy et al. 2018; Battaglia and Kollias 2019) begins by combining the observed reflectivities to form the observed absorption coefficient

$$
\begin{aligned}
\gamma\left(r_{1}, r_{2}, f\right)= & \frac{1}{2 R} \ln \left[\frac{Z_{\mathrm{obs}}\left(r_{1}, f\right)}{Z_{\mathrm{obs}}\left(r_{2}, f\right)}\right] \\
= & \frac{1}{2 R} \ln \left[\frac{Z\left(r_{1}, f\right)}{Z\left(r_{2}, f\right)}\right]+\frac{1}{R} \int_{r_{1}}^{r_{2}} d r^{\prime}\left[\beta_{g}\left(r^{\prime}, f\right)\right. \\
& \left.+\beta_{h}\left(r^{\prime}, f\right)\right],
\end{aligned}
$$

where $r_{2}-r_{1}=R$. Next, we write $\beta_{g}(r, f)=\rho_{v}(r) \kappa_{v}(r$, $f)+\beta_{g, d}(r, f)$, where $\rho_{v}$ is the water vapor density, $\kappa_{v}$ is the mass extinction cross section including contributions from all $\mathrm{H}_{2} \mathrm{O}$ absorption lines (i.e., the continuum contribution), and $\beta_{g, d}$ is the dry-air absorption coefficient. In this work, we calculate gaseous absorption parameters using the millimeter-wave propagation model from the EOS Microwave Limb Sounder (Read et al. 2004). Making the assumption that the spatial variation of $\kappa_{v}$ and $\beta_{g, d}$ is small on the scale $R$, we replace their values in the integrand in Eq. (5) with those at the midpoint $\bar{r}=\left(r_{1}+r_{2}\right) / 2$ and evaluate the integral to yield

$$
\gamma\left(r_{1}, r_{2}, f\right)=\alpha\left(r_{1}, r_{2}, f\right)+\bar{\rho}_{v} \kappa_{v}(\bar{r}, f)+\beta_{g, d}(\bar{r}, f)+\bar{\beta}_{h}(f),
$$

where $\alpha=(2 R)^{-1} \ln \left[Z\left(r_{1}, f\right) / Z\left(r_{2}, f\right)\right]$ and $\bar{\rho}_{v}$ and $\bar{\beta}_{h}$ are the average values of the respective quantities between $r_{1}$ and $r_{2}$. Next, we perform a Taylor expansion of hydrometeor scattering quantities $Z$ and $\beta_{h}$, which involve integrations of single particle scattering parameters over a DSD, and retain only the first-order frequency dependence because of the small fractional bandwidths used for DAR systems $(1 \%-10 \%)$. Note that the equivalent parameters for DIAL systems can be taken to be independent of frequency because of the extremely small fractional bandwidths involved.

Thus, the standard DAR retrieval amounts to performing a least squares fit of the function

$$
\hat{\gamma}=a_{1}+a_{2} \times\left(f-f_{1}\right)+a_{3} \times \kappa_{v}(\bar{r}, f)+\beta_{g, d}(\bar{r}, f)
$$

to the data, with the fitting parameters having the physical interpretations $a_{1}=\alpha\left(r_{1}, r_{2}, f_{1}\right)+\bar{\beta}_{h}\left(f_{1}\right), a_{2}=(\partial / \partial f)(\alpha+$ $\left.\bar{\beta}_{h}\right)\left.\right|_{f_{1}}$, and $a_{3}=\bar{\rho}_{v}$, where $f_{1}$ is a reference frequency within the transmitted band. Seemingly, then, measurements of $Z_{\mathrm{obs}}$ at three different frequencies should suffice to fully determine these coefficients and retrieve unbiased humidity estimates. However, if $\kappa_{v}$ is nearly linear across the range of frequencies used, then $a_{2}$ and $a_{3}$ become degenerate fitting parameters, and the choice of including a linear term leads to an inflation of the estimated humidity variance. Clearly, the measurement in this case cannot distinguish between frequency-dependence due to humidity and hydrometeor scattering. Hence, DAR retrievals will be most accurate when $\kappa_{v}$ exhibits strong curvature with respect to frequency. Unfortunately, however, $\kappa_{v}$ has very little curvature across the transmission band used in this work, and so utilizing the standard DAR retrieval would require setting $a_{2}=0$. The frequencydependent hydrometeor terms would then enter directly as a bias. As shown in the next section, we attempt to limit these biases by retrieving the entire humidity profile in a single regularized least squares minimization routine. 


\section{b. Regularized least squares approach}

In this section, we present a new retrieval algorithm for the case of two transmitted frequencies, $f_{1}$ and $f_{2}$, in which the entire humidity profile and corresponding covariance matrix are estimated in a single least squares minimization procedure, in contrast to previous approaches that iteratively retrieve the humidity at each height (Roy et al. 2018; Battaglia and Kollias 2019). This approach has the additional advantage of being amenable to regularization methods that constrain the form of the retrieved profile. The extension of this method to arbitrarily many frequencies is straightforward.

We begin the formulation of the least squares retrieval by defining the measurement vector $\mathbf{y}=\left[\mathbf{y}_{1}, \mathbf{y}_{2}\right]$, where $\left[\mathbf{y}_{j}\right]_{i}=\ln \left[Z_{\text {obs }}\left(r_{i}, f_{j}\right) / Z_{0}\right]$ and $\mathbf{r}=\left[r_{1}, r_{2}, \ldots, r_{N_{r}}\right]$ is the collection of radar ranges for which the reflectivity measurements at all frequencies have SNR greater than some threshold value. For the in-cloud humidity profile retrievals presented in this work, we set this threshold to 1. The measurement vector therefore has length $2 N_{r}$. The choice of using the logarithm of reflectivity, and not linear units, in the measurement vector ensures that the forward model is linear in the humidity, and is justified so long as the relative error of the $Z_{\text {obs }}$ measurement is small. For the measurements presented in this work, this condition is satisfied due to the large number of pulses $N=2000$ averaged at each frequency, with a relative error of $0.25 \%$ for an SNR of 1 . The resulting measurement covariance matrix is block diagonal, since the measurement errors at different frequencies are uncorrelated, with $\mathbf{S}_{y}=\operatorname{diag}\left(\mathbf{S}_{\mathbf{y}_{1}}, \mathbf{S}_{\mathbf{y}_{2}}\right)$. To determine the form of each individual block, we first calculate the covariance matrix for the reflectivity vector $\mathbf{z}=\left[\mathbf{Z}_{\text {obs }}\left(f_{1}\right)\right.$, $\mathbf{Z}_{\text {obs }}\left(f_{2}\right)$ ], and then transform to the measurement space according to $\mathbf{S}_{y}=\mathbf{J}_{z} \mathbf{S}_{z} \mathbf{J}_{z}^{\mathrm{T}}$, where $\mathbf{S}_{z}=\operatorname{diag}\left[\mathbf{S}_{Z}\left(f_{1}\right)\right.$, $\left.\mathbf{S}_{Z}\left(f_{2}\right)\right]$ is the covariance matrix for $\mathbf{z}$ and $\mathbf{J}_{z}$ is the Jacobian of $\mathbf{y}$ with respect to $\mathbf{z}$. The individual covariance matrices for observed reflectivity measurements are themselves tridiagonal, with

$$
\begin{aligned}
{\left[\mathbf{S}_{Z}\left(f_{i}\right)\right]_{j k}=} & C^{2}\left(f_{i}\right) r_{j}^{2}\left\{r_{j}^{2} \operatorname{var}\left[P_{e}\left(r_{j}\right)\right] \delta_{j k}\right. \\
& \left.+r_{k}^{2} \operatorname{cov}\left[P_{e}\left(r_{j}\right), P_{e}\left(r_{k}\right)\right]\left(\delta_{j, k+1}+\delta_{j, k-1}\right)\right\},
\end{aligned}
$$

where the power variance and covariance terms are defined in Eqs. (3) and (4), and $\boldsymbol{\delta}$ is the Kronecker delta. Note that if adjacent elements of the measurement vector do not correspond to adjacent radar range bins (i.e., if $\left|r_{j}-r_{k}\right| \neq \Delta r$ ), then the off-diagonal term is zero.

Next, we must define the state vector $\mathbf{x}$ and forward $\operatorname{model} \mathbf{F}(\mathbf{x}, \mathbf{b})$, where $\mathbf{b}$ is a vector of parameters that are input to the forward model, but not retrieved. A full radiative model for the beam propagation would need to account for spatial distribution of the cloud microphysical properties (e.g., DSD parameters) as well as atmospheric parameters of temperature, pressure, and humidity. However, the DAR approach is powerful because it is largely insensitive to all of these details except for the humidity. Thus, we want a forward model that amounts to a fully determined linear system of equations, as is the case for the standard differential absorption retrieval [Eq. (7)]. We accomplish this by choosing the state vector elements to be $\mathbf{x}=\left[\mathbf{x}_{1}, \mathbf{x}_{2}\right]$, where $\left[\mathbf{x}_{1}\right]_{i}=\ln \left\{\left[\mathbf{Z}_{r}\left(f_{1}\right)\right]_{i} / Z_{0}\right\}$ and $\mathbf{Z}_{r}\left(f_{1}\right)=\left[Z_{r}\left(r_{1}, f_{1}\right)\right.$, $\left.Z_{r}\left(r_{2}, f_{1}\right), \ldots, Z_{r}\left(r_{N}, f_{1}\right)\right]$ consists of the retrieved reflectivities at one of the transmitted frequencies and includes attenuation from hydrometeors, but not gaseous attenuation, and $\mathbf{x}_{2}=\boldsymbol{\rho}_{v}$ consists of the humidity values to be retrieved at ranges $\mathbf{r}_{\rho}=\left[r_{\rho, 1}, r_{\rho, 2}, \ldots, r_{\rho, M}\right]$.

Because of the potential sparsity of the observed reflectivity profile, the definition of the retrieved humidity profile $\boldsymbol{\rho}_{v}$ and its corresponding range axis requires some care. To maximally utilize the information in the measurements, we want the retrieval to both profile inside of continuous cloud volumes with resolution $R$ and return IWV values in between cloud boundaries as well as between the radar and first cloud signal. Previous DAR retrieval methods based on fixed differencing in space of measurements separated by $R$ lose the ability to extract information between cloud layers with large separations. As we will see, this problem is naturally avoided when using the least squares solution approach, since the entire humidity profile is retrieved from a minimization problem in which the forward model interpolates between all humidity points, and not just those separated by $R$. Anticipating that we will want to build up time series of humidity profiles with regular gridding in space, we enforce that the humidity positions be of the form $r_{\rho, i}=n_{i} R$ for some integer $n_{i}$. Then, given a measurement range vector $\mathbf{r}$ with maximum value $r_{\text {max }}$, we form $\mathbf{r}_{\rho}$ by retaining all values $n_{i}$ for which there is at least one element of $\mathbf{r}$ in the interval $\left[\left(n_{i}-1 / 2\right) R,\left(n_{i}+1 / 2\right) R\right)$. Additionally, we always include the surface point $n_{i}=0$ as the first entry in $\mathbf{r}_{\rho}$.

The first step in constructing the forward model is to interpolate the lower-resolution humidity profile to the radar range resolution $\Delta r$, giving a new humidity vector $\tilde{\boldsymbol{\rho}}$ with range axis $\tilde{\mathbf{r}}=[0, \Delta r, 2 \Delta r, \ldots]$. Next, we use profiles of temperature and pressure at the same $\Delta r$ resolution to calculate the resulting gasabsorption optical depth vector $\tilde{\boldsymbol{\tau}}_{g}=\left[\tilde{\boldsymbol{\tau}}_{g}\left(f_{1}\right), \tilde{\boldsymbol{\tau}}_{g}\left(f_{2}\right)\right]$ according to

$$
\tilde{\boldsymbol{\tau}}_{g, i}\left(f_{j}\right)=\tilde{\boldsymbol{\tau}}_{g, i-1}\left(f_{j}\right)+\Delta r\left[\beta_{g, d}\left(\tilde{r}_{i-1}, f_{j}\right)+\tilde{\boldsymbol{\rho}}_{i-1} \kappa_{v}\left(\tilde{r}_{i-1}, f_{j}\right)\right],
$$


with $\tilde{\boldsymbol{\tau}}_{g, 0}=0$. Note that the gas absorption dependence on temperature and pressure are implicit in the $\tilde{r}$ dependence. For the retrievals presented in section 4, we use the radiosonde surface measurements and assume hydrostatic balance with a $6.5^{\circ} \mathrm{C} \mathrm{km}^{-1}$ lapse rate to calculate the temperature and pressure profiles. Last, the calculated optical depth vector is then masked to only include values at the measured range positions $\mathbf{r}$, yielding the vector $\tau_{g}$ of length $2 N_{r}$. The recursive relationship in Eq. (9) can be cast as a matrix equation of the form

$$
\boldsymbol{\tau}_{g}=\mathbf{T} \boldsymbol{\rho}_{v}+\boldsymbol{\tau}_{g, d}
$$

which will be necessary for the matrix inversion solution to the regularized least squares problem. Here $\boldsymbol{\tau}_{g, d}$ is a constant vector of dry air optical depths, and $\mathbf{T}$ is a $2 N_{r} \times M$ matrix.

The forward model is then defined as $\mathbf{F}(\mathbf{x}, \mathbf{b})=[\mathbf{F}(\mathbf{x}, \mathbf{b}$, $\left.\left.f_{1}\right), \mathbf{F}\left(\mathbf{x}, \mathbf{b}, f_{2}\right)\right]$, where

$$
\begin{aligned}
& {\left[\mathbf{F}\left(\mathbf{x}, \mathbf{b}, f_{1}\right)\right]_{i}=\ln \left[Z_{r}\left(r_{i}, f_{1}\right) / Z_{0}\right]-2 \tau_{g, i}\left(f_{1}\right),} \\
& {\left[\mathbf{F}\left(\mathbf{x}, \mathbf{b}, f_{2}\right)\right]_{i}=\ln \left[d_{i} Z_{r}\left(r_{i}, f_{1}\right) / Z_{0}\right]-2 \tau_{g, i}\left(f_{2}\right),}
\end{aligned}
$$

and $d_{i}$ is a fixed scaling factor that accounts for differential backscattering coefficients between the two frequencies. Using Eq. (10) and the definition of $\mathbf{x}$, we can write the forward model as

$$
\mathbf{F}(\mathbf{x}, \mathbf{b})=\mathbf{K} \mathbf{x}+\mathbf{b},
$$

where

$$
\mathbf{K}=\left[\begin{array}{c|c}
\mathbf{I}_{N_{r}} & \mathbf{T} \\
\mathbf{I}_{N_{r}} & \mathbf{T}
\end{array}\right]
$$

is defined in block form, $\mathbf{I}_{N_{r}}$ is the $N_{r} \times N_{r}$ identity matrix, and $\mathbf{b}$ includes both the $d_{i}$ and $\boldsymbol{\tau}_{g, d}$ terms. For this work, we choose the same value $d$ for all $d_{i}$, informed by Lorenz-Mie calculations of liquid hydrometeor scattering (Bohren and Huffman 2004). This is equivalent to assuming that the DSD characteristic diameter is constant in space. The range of $d$ values for different DSDs is shown in Fig. 2, where we assume a modified gamma distribution as in Roy et al. (2018) and vary the shape parameter $\nu$ from 1 to 4 to explore the sensitivity to the assumed DSD shape. Focusing on the $\nu=4$ case, which is a typical value used for nonprecipitating clouds, we see that the range of characteristic diameters over which $\mathbf{b}$ varies the most is $10-100 \mu \mathrm{m}$. To be conservative, we pick for our value of $\mathbf{b}$ the average of the two limits in Fig. 2, or $d=0.9$, and assign a systematic uncertainty of $\sigma_{d}=0.1$.

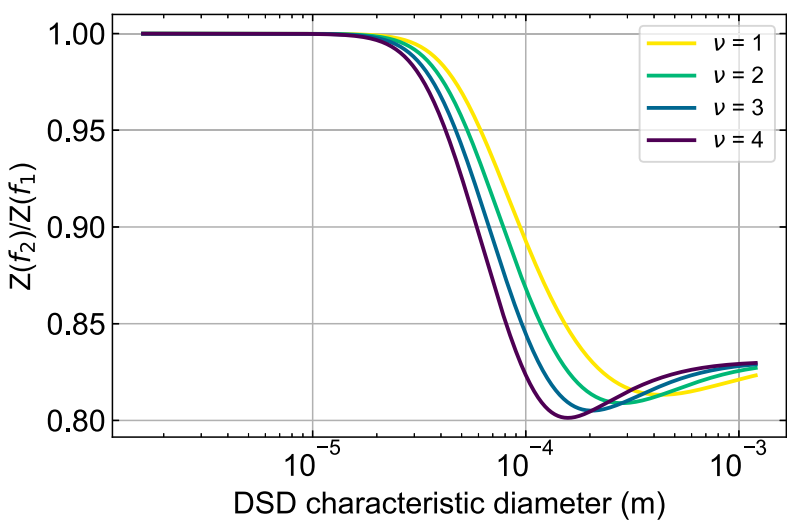

FIG. 2. Ratio of reflectivity at $f_{2}=174.8 \mathrm{GHz}$ and $f_{1}=167 \mathrm{GHz}$ for liquid water spheres at $280 \mathrm{~K}$, integrated over a modified gamma DSD for four different shape parameters $\nu$.

It is important to note that for DAR humidity profiling, systematic biases stemming from frequencydependent backscattering would only arise if $d_{i}$ varies in range. In the context of this retrieval, then, it is clear that our choice of constant $d$ will not correct for these potential biases. However, this formulation gives us a way to propagate our uncertainty about these effects into our eventual estimates of humidity. Specifically, imperfect knowledge of the forward model parameters b can be represented by a systematic error covariance matrix in the measurement space according to (Rodgers 2000) $\mathbf{S}_{e}=\mathbf{J}_{\mathbf{b}} \mathbf{S}_{\mathbf{b}} \mathbf{J}_{\mathbf{b}}^{\mathrm{T}}$, where $\mathbf{S}_{\mathbf{b}}=\sigma_{d}^{2} \mathbf{I}_{N_{r}}$ and $\mathbf{J}_{\mathbf{b}}$ is the $2 N_{r} \times$ $N_{r}$ Jacobian of the forward model with respect to $\mathbf{b}$. It is easy to show that the resulting $\mathbf{S}_{e}$ is a $2 N_{r} \times 2 N_{r}$ diagonal matrix with $\left[\mathbf{S}_{e}\right]_{i i}-\left(\sigma_{d} / d\right)^{2}$ for $i>N_{r}$, and $\left[\mathbf{S}_{e}\right]_{i i}=0$ for $i \leq N_{r}$. It may appear that, because $Z_{\mathrm{obs}}(r, f) \infty \mathrm{C}(f)$, the measurement error ought to include uncertainty in the determination of $\mathrm{C}(f)$. However, a miscalibrated value of $\mathrm{C}(f)$ will only affect the water vapor measurement between the radar and the first echo range, with subsequent retrieved humidity values being completely insensitive to this factor. Therefore, we do not include calibration uncertainty in the measurement covariance analysis, and will discuss the related column-integrated water vapor issues in the next section.

Finally, in order to mitigate the humidity biases that arise from the linear term in Eq. (7), we implement a regularization term. We begin by noting that these biases often occur over short spatial scales, for instance due to the differential backscattering at a boundary between intense and light precipitation, or between precipitation and a cloud base, and thus tend to produce large, unphysical gradients of water vapor in the retrieved profile. A natural regularization term to utilize, then, is one that penalizes sharp gradients in the retrieved state. For this term, we choose the sum of squared discrete derivatives 
of the humidity vector, which can be written as a quadratic form

$$
\mathbf{x}^{\mathrm{T}} \mathbf{A} \mathbf{x}=\delta_{\rho}^{-2} \sum_{i=1}^{M-1}\left(\frac{\rho_{i+1}-\rho_{i}}{r_{\rho, i+1}-r_{\rho, i}}\right)^{2}
$$

for some matrix $\mathbf{A}$, where $\delta_{\rho}$ is some chosen scale for penalized humidity gradients, and is set to $10 \mathrm{~g} \mathrm{~m}^{-3} \mathrm{~km}^{-1}$ in this work. The inverse algorithm is then the solution to a least squares minimization problem,

$$
\hat{\mathbf{x}}=\underset{\mathbf{x}}{\operatorname{argmin}} \mathscr{C}(\mathbf{x}),
$$

where the cost function is given by

$$
\begin{aligned}
\mathscr{C}(\mathbf{x})= & {[\mathbf{y}-\mathbf{F}(\mathbf{x}, \mathbf{b})]^{\mathrm{T}}\left(\mathbf{S}_{y}+\mathbf{S}_{e}\right)^{-1}[\mathbf{y}-\mathbf{F}(\mathbf{x}, \mathbf{b})] } \\
& +\lambda_{\text {reg }} \mathbf{x}^{\mathrm{T}} \mathbf{A} \mathbf{x},
\end{aligned}
$$

and $\lambda_{\text {reg }} \geq 0$ is a dimensionless parameter that determines how severely to penalize humidity gradients. To implement the gradient regularization scheme, we need to find the symmetric matrix $\mathbf{A}$ that satisfies Eq. (14). One can show that $\mathbf{A}$ is block diagonal and of the form $\mathbf{A}=\operatorname{diag}\left(\mathbf{0}_{N_{r}}, \delta_{\rho}^{-2} \mathbf{D}^{\mathrm{T}} \boldsymbol{\Delta}_{r}^{-2} \mathbf{D}\right)$, where the first block is a $N_{r} \times N_{r}$ matrix of zeros, [D] $]_{i j}=\delta_{i, j-1}-\delta_{i, j}$ is a $(M-1) \times$ $M$ finite differencing matrix, and $\left[\boldsymbol{\Delta}_{r}\right]_{i j}=\delta_{i, j}\left(r_{\rho, i+1}-r_{\rho, i}\right)$ is a $(M-1) \times(M-1)$ diagonal matrix. We set the regularization parameter $\lambda_{\text {reg }}=1$, which amounts to a conservative penalty of gradients on the scale $\delta_{\rho}$. This gradient regularization method is similar in nature to applying a spatial low-pass filter to the retrieved humidity profile, but performs the smoothing as part of a single least squares optimization routine, works for nonregularly spaced points, and naturally allows for uncertainty quantification in the retrieved, smoothed quantities. [An example scenario where this profilesmoothing approach is particularly effective is shown in Fig. 4 (second row, left panel).] Here the red curve represents the standard two-frequency DAR retrieval employed in Roy et al. (2018), which exhibits significant biases due to spatially varying hydrometeor properties, while the regularized least squares approach successfully mitigates these effects. We note that the value of $\delta_{\rho}$ is chosen as a compromise between limiting biases that appear as sharp humidity gradients and avoiding an overly constraining low-pass filter, and that using $10 \mathrm{~g} \mathrm{~m}^{-3} \mathrm{~km}^{-1}$ results in good agreement between the median retrieval error and median bias with respect to radiosonde measurements (Fig. 5b). Because of this choice, the retrieval will smooth out real humidity variations that are on the scale of $\delta_{\rho}$ or larger.
Because the forward model is linear in the state vector, the minimization problem [Eq. (15)] can be solved by means of a matrix inversion,

$$
\hat{\mathbf{x}}=\left[\mathbf{K}^{\mathrm{T}}\left(\mathbf{S}_{y}+\mathbf{S}_{e}\right)^{-1} \mathbf{K}+\lambda_{\text {reg }} \mathbf{A}\right]^{-1} \mathbf{K}^{\mathrm{T}}\left(\mathbf{S}_{y}+\mathbf{S}_{e}\right)^{-1}(\mathbf{y}-\mathbf{b}) .
$$

Furthermore, the retrieved state covariance matrix is given by the Hessian of $\mathscr{C}(\mathbf{x})$,

$$
\hat{\mathbf{S}}_{x}=\left[\mathbf{K}^{\mathrm{T}}\left(\mathbf{S}_{y}+\mathbf{S}_{e}\right)^{-1} \mathbf{K}+\lambda_{\text {reg }} \mathbf{A}\right]^{-1} .
$$

We note here that the regularization term is not introduced because of ill-posedness of the unregularized problem, as is the case with the introduction of the prior distribution in optimal estimation (Rodgers 2000), but rather to constrain the form of the retrieved profile. From the form of $\hat{\mathbf{S}}_{x}$ in Eq. (18), it becomes apparent that inclusion of the systematic error covariance matrix $\mathbf{S}_{e}$ can be viewed as effectively inflating the measurement variance. To get a sense of the scale of this variance inflation, we utilize the fact that the diagonal elements of $\mathbf{S}_{y}$ are equal to the squared relative error of the corresponding echo power measurement, $\operatorname{var}\left[P_{e}(r, f)\right] / P_{e}^{2}(r, f)$, and those for $\mathbf{S}_{e}$ are either zero or equal to $\left(\sigma_{d} / d\right)^{2}$. Thus, using Eq. (3) for the measurement relative error with $N=2000$ pulses, we see that this approach amounts to an increase in variance by a factor of about 20 for high-SNR measurements, and by a factor of about 4 for measurements with SNR $=1$.

\section{c. Column-integrated water vapor}

The IWV measurement between the surface and an elevated cloud base is an analogous measurement to future column measurements made from an airborne platform between the radar and the surface. These measurements are of interest for any DAR because in the absence of clouds, the surface return is the only radar measurement acquired. For the case of a single-range measurement at two frequencies, one can solve for the IWV between the radar and range $r$ :

$$
\begin{aligned}
\mathrm{IWV}= & \frac{1}{2\left\langle\Delta \kappa_{v}\right\rangle}\left\{\ln \left[\frac{P_{e}\left(r, f_{1}\right)}{P_{e}\left(r, f_{2}\right)}\right]+\ln \left[\frac{C\left(f_{1}\right)}{C\left(f_{2}\right)}\right]\right. \\
& \left.+\ln \left[\frac{Z\left(r, f_{2}\right)}{Z\left(r, f_{1}\right)}\right]-2 \int_{0}^{r} d r^{\prime} \Delta \beta_{h}\left(r^{\prime}\right)\right\},
\end{aligned}
$$

where IWV $=\int_{0}^{r} d r^{\prime} \rho_{v}\left(r^{\prime}\right)$,

$$
\left\langle\Delta \kappa_{v}\right\rangle=\frac{\int_{0}^{r} d r^{\prime} \rho_{v}\left(r^{\prime}\right) \Delta \kappa_{v}\left(r^{\prime}\right)}{\int_{0}^{r} d r^{\prime} \rho_{v}\left(r^{\prime}\right)},
$$


$\Delta \kappa_{v}(r) \equiv \kappa_{v}\left(r, f_{2}\right)-\kappa_{v}\left(r, f_{1}\right)$, and $\Delta \beta_{h}(r) \equiv \beta_{h}\left(r, f_{2}\right)-$ $\beta_{h}\left(r, f_{1}\right)$. The first term in Eq. (19) will have an associated random error, while the remaining three may introduce systematic error. In this work, we only utilize measurements with negligible hydrometeor content between the surface and $r$ and therefore can neglect the last term in this equation dealing with differential absorption from hydrometeors. Using $\left\langle\Delta \kappa_{v}\right\rangle \approx 0.25 \mathrm{~dB} \mathrm{~km}^{-1} \mathrm{~g}^{-1} \mathrm{~m}^{3}$, which is representative of lower-tropospheric conditions, we see from Eq. (19) that errors in the individual bias terms of $1 \mathrm{~dB}$ lead to a IWV bias of $2 \mathrm{~mm}$.

To assess VIPR's ability to accurately measure columnintegrated water vapor, we perform a set of retrievals in which the surface-to-cloud-base IWV is the only humidity quantity retrieved. We assume that the water vapor density decays exponentially in the vertical with a scale height of $2 \mathrm{~km}$, which is consistent with previous measurements at the ARM SGP site (Turner et al. 2001). For these retrievals, we lower the nominal SNR threshold to 0.5 , and in some cases to 0.25 if there are no returns with SNR $>0.5$, allowing for IWV measurements using cloud layers with weak reflectivities, and utilize reflectivity measurements within a distance $R$ of the bottom cloud edge. The main reason for performing a separate set of IWV retrievals, as opposed to using the corresponding value from the profiling retrieval detailed in the previous section, is that the latter occurs on a fixed, equally spaced vertical grid. Therefore, depending on where the cloud base falls within a vertical grid cell of size $R$, the profiling retrieval will utilize a different number of reflectivity measurements to estimate the surface-to-cloud-base IWV, and thus have variable sensitivity. Furthermore, we change the scaling factor for differential backscattering between $f_{1}$ and $f_{2}$ from $d=0.9$ to $d=1$, since we expect scattering to be of Rayleigh character near the cloud edge where reflectivities are very weak. Unlike the case of in-cloud profiling, where taking the ratio of reflectivities at two different ranges makes the measurement insensitive to the value of $d$, incorrect specification of this parameter biases the IWV estimate [see Eq. (19)]. We take a practical approach to determining the calibration ratio $C\left(f_{1}\right) / C\left(f_{2}\right)$, and choose the value that gives zero average IWV bias with respect to the ARM instrument ground truth. Therefore, average biases resulting from our choice of $d$ and from hydrometeor absorption are implicitly included in the determined value of $C\left(f_{1}\right) / C\left(f_{2}\right)$.

\section{Results and discussion}

\section{a. In-cloud humidity profiles}

For validation of VIPR's in-cloud profiling capabilities, we utilize cloud reflectivity measurements acquired on multiple days within the 2-14 April 2019 window. Three example datasets with significant spatiotemporal cloud coverage are presented in Fig. 3. We present the observed reflectivity measurements at $f_{1}=167 \mathrm{GHz}$ for which SNR $>1$. These $2 \mathrm{D}$ plots have the nominal radar time resolution of about $5 \mathrm{~s}$, and range resolution of $15 \mathrm{~m}$. For these and all subsequent plots, the height is referenced to mean sea level, with the ground level at ARM being $0.3 \mathrm{~km}$. While there is no near-range dead zone in FMCW radar, we do not use reflectivity measurements within the first $100 \mathrm{~m}$, because these correspond to the antenna near field and the calibration measurements were performed in the far field. Depending on the level of precipitation at the surface, VIPR is either positioned outside pointing at the zenith, or just inside the facility pointing between $20^{\circ}$ and $30^{\circ}$ off zenith. The measurements presented in Fig. 3 account for these adjustments in pointing angle.

There are two features in the reflectivity datasets to highlight. First, there is a signature of the melting layer via an abrupt reflectivity change at a persistent height on each of the three days once precipitation is initiated, as we would expect for the cold rain process within the stratiform anvils associated with deep convective clouds. Second, there is a clear impact on the maximum detectable range in the presence of precipitation, due to the greatly increased hydrometeor absorption experienced by the radar beam. Additionally, note that because the PBL humidity on 13 April is less than half that on the previous days, the upper-tropospheric clouds appear to have a larger reflectivity as the beam absorption from water vapor is greatly reduced. In fact, for both of these reasons it is clear why DAR measurements from an airborne or spaceborne platform will offer a substantial increase in sampling precipitating clouds, as the hydrometeors and low humidity levels aloft will not extinguish the beam before it reaches the strongly reflective rain drops in the lower levels.

The lower panels in Fig. 3 are the retrieved, in-cloud humidity fields from the reflectivity measurements at 167 and $174.8 \mathrm{GHz}$ (the latter are not shown). We utilize a humidity profile resolution of $R=180 \mathrm{~m}$, which is a factor of 12 larger than the reflectivity resolution. The launch times of radiosondes from the ARM central facility are depicted with black dashed lines. Note that in-cloud humidity can only be retrieved where there are measurements at both frequencies, and since $f_{2}$ experiences more atmospheric absorption than $f_{1}$, the spatiotemporal coverage of humidity is slightly less than that for reflectivity at $f_{2}$.

A close scrutiny of the corresponding reflectivity and humidity plots in Fig. 3 reveals that some regions exhibit a correlation between spatial boundaries of $\mathrm{dB} Z$ 
(a)
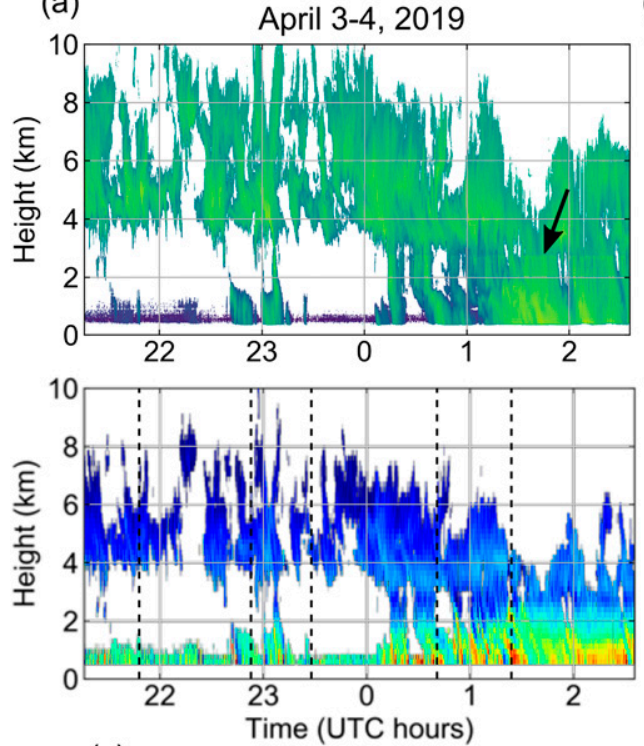

(b) April 6, 2019
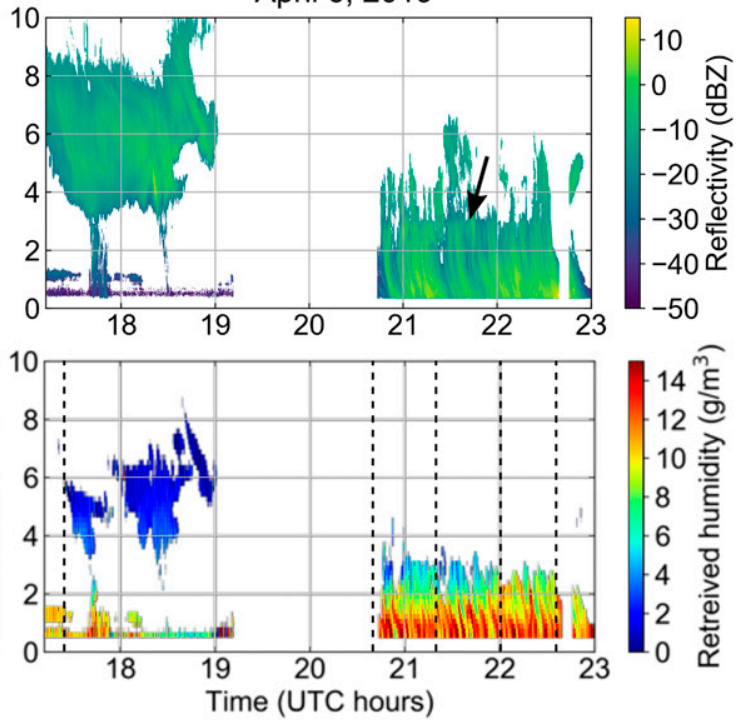

April 13, 2019
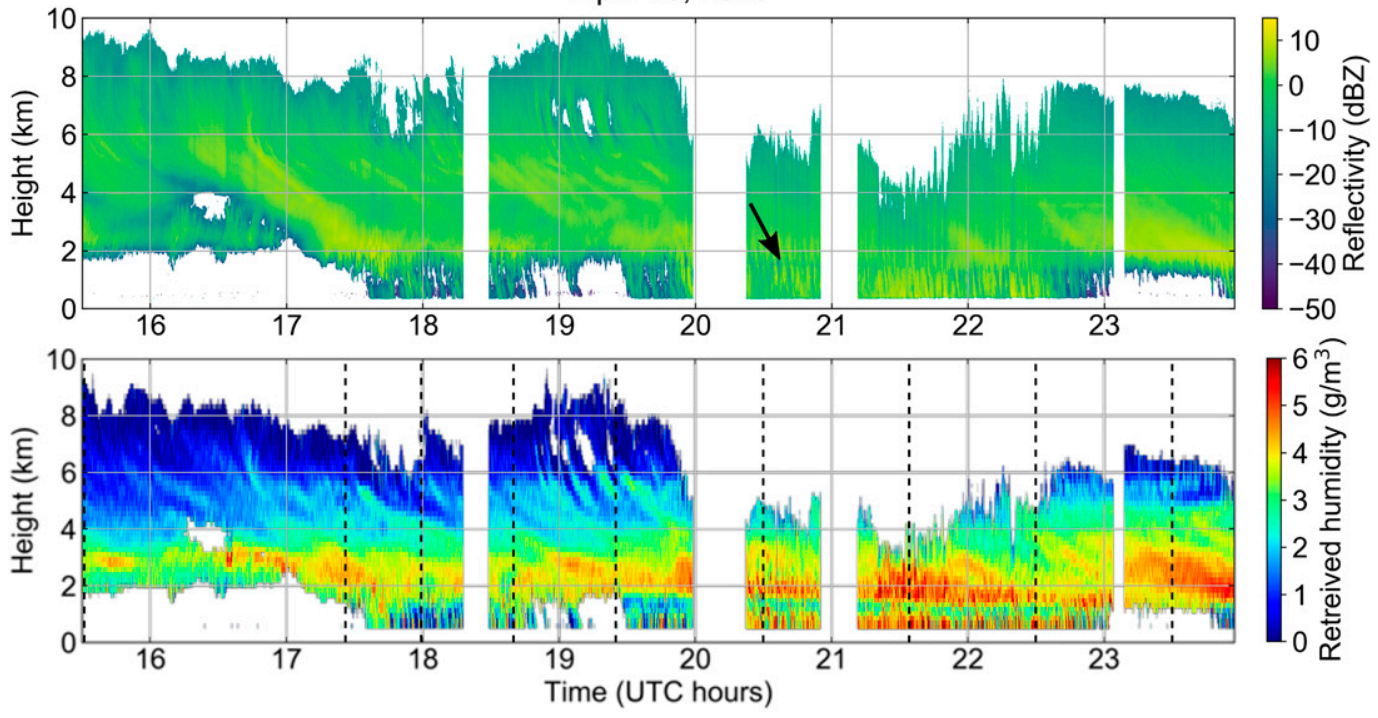

FIG. 3. Observed cloud reflectivities at $167 \mathrm{GHz}$ and corresponding retrieved humidity profiles for three different days. Dashed vertical lines correspond to radiosonde launch times. Time periods with no reflectivity measurements are either cloud-free scenes or periods when the radar was temporarily not acquiring data. Arrows indicate the melting-layer position.

and changes in $\rho_{v}$. For instance, observe the marked depression in humidity correlated with and just below the melting layer position in periods with precipitation in Fig. 3c, or the temporal variations of magnitude $>30 \%$ that are correlated with distinct bands of precipitation in the latter part of Fig. 3b. Comparisons of retrieved humidity profiles at the nominal $5 \mathrm{~s}$ radar time resolution with radiosonde measurements show that these variations are not physical. In fact, such features are a manifestation of the systematic bias from differential hydrometeor reflectivity, and are the reason for implementing the gradient-penalty regularization scheme. Specifically, if the characteristic diameter of the DSD changes quickly in space, we expect a large value of the second term in Eq. (7), which can significantly bias the retrieved value of $\rho_{v}$. For instance, using the Lorenz-Mie calculations in Fig. 2 for $\nu=4$, if the characteristic diameter varies from 20 to $60 \mu \mathrm{m}$ over $180 \mathrm{~m}$, there would be a resulting DAR humidity bias of $5 \mathrm{~g} \mathrm{~m}^{-3}$.

While these particular variations in the retrieved humidity are not random in nature, we can reduce their effect on the accuracy of the measurement further by 
averaging vertical $\rho_{v}$ profiles in time. This will help to smooth out variations of the type seen in Fig. 3b, but not that associated with the melting layer in Fig. 3c. While this time-averaging procedure decreases the effective DAR $\rho_{v}$ time resolution, the situation will be much different when VIPR is deployed from an airborne platform. In this case, the fast aircraft motion allows VIPR to sample the spatial heterogeneities of a cloud scene much faster and therefore should permit a humidity measurement time resolution near the nominal value of $5 \mathrm{~s}$. This is clear from low level of random noise evident in the humidity fields in Fig. 3, and from the fact that the error bars in the DAR-retrieved humidity are dominated by the introduction of the systematic error term $\mathbf{S}_{e}$, and have little contribution from the random measurement noise except where the SNR is low.

Because the time-varying systematic biases in $\rho_{v}$ are not random, we must account for covariance between adjacent time values in determining the resulting uncertainty. The recipe we use to compute time-averaged $\rho_{v}$ profiles involves first partitioning the $2 \mathrm{D}$ humidity fields into segments of duration $T_{\mathrm{avg}}=10 \mathrm{~min}$. Because of the sparsity of DAR datasets, it is often the case that there are many missing data points in each segment. Therefore, we require that each segment have a minimum of 10 measurements in order to record a final averaged humidity value. Given a set of humidity measurements at a given height $z_{i}$ and between time $t$ and $t+T_{\text {avg }},\left\{\rho_{v}\left(z_{i}, t_{1}\right), \rho_{v}\left(z_{i}, t_{2}\right), \ldots, \rho_{v}\left(z_{i}, t_{M}\right)\right\}$, we compute the time-average mean and variance from $\bar{\rho}_{v}\left(z_{i}\right)=M^{-1} \sum_{j=1}^{M} \rho_{v}\left(z_{i}, t_{j}\right)$ and

$$
\operatorname{var}\left[\bar{\rho}_{v}\left(z_{i}\right)\right]=M^{-2} \sum_{j, k=1}^{M}\left[\mathbf{S}_{\rho_{v}, i}\right]_{j k},
$$

where the $\rho_{v}$ time covariance matrix has elements $\left[\mathbf{S}_{\rho_{v},},\right]_{j k}=$ $\sigma_{\rho_{v}}\left(z_{i}, t_{j}\right) \sigma_{\rho_{v}}\left(z_{i}, t_{k}\right) \exp \left(-\left|t_{j}-t_{k}\right| / \tau_{\text {corr }}\right), \sigma_{\rho_{v}}\left(z_{i}, t_{k}\right)$ is the uncertainty returned by the retrieval algorithm, and we set the exponential time-correlation constant to $\tau_{\text {corr }}=1 \mathrm{~min}$. Note that because of the imposed correlations in the time-averaging procedure, the improvement in humidity precision with averaging time is less than that for uncorrelated measurement averaging $\sqrt{T_{\text {avg }} / \Delta t}$, where $\Delta t=5 \mathrm{~s}$ is the nominal temporal resolution.

The resulting comparisons of coincident DAR and RS humidity observations are presented in Fig. 4. The only profiles excluded from this figure are those in which the DAR profile spans less than $2 \mathrm{~km}$. In these plots, the error bars correspond to the square root of the timeaveraged humidity variance in Eq. (21) (i.e., $1 \sigma$ error). These are the first-ever validated measurements of water vapor inside of clouds using an active remote sensing technique, and show the capability of VIPR to accurately profile in-cloud humidity not only in the PBL, but throughout the troposphere.

While the coincident DAR/RS measurements make up a useful dataset for evaluating VIPR's humidity accuracy, we would like to be able to use the remaining 10-min-averaged profiles as well. Therefore, we linearly interpolate the RS profiles in time at each height to the DAR temporal resolution $T_{\text {avg }}$, and note the observation that RS humidity profiles do not change appreciably in the intervals between launches (usually $0.5-2 \mathrm{~h}$ ). Furthermore, we smooth the RS profiles in height to make the effective resolution equal to that of the DAR profiles by performing a convolution with a box of length $R$. The resulting comparison between remotely sensed and in situ humidity is presented in Fig. 5. The scatterplot in Fig. 5a has an associated correlation coefficient $r=0.96$ and RMSE of $0.8 \mathrm{~g} \mathrm{~m}^{-3}$. The corresponding cumulative distribution of the absolute value of DAR humidity bias relative to the interpolated RS profiles is shown in Fig. 5b. Note that we describe bias in absolute terms (i.e., in units of $\mathrm{g} \mathrm{m}^{-3}$ ) and not in terms of relative error, because the sensitivity of a differential absorption measurement is independent of the magnitude of $\rho_{v}$ (Roy et al. 2018). Therefore, this approach is a more appropriate assessment of the DAR accuracy. We find that biases are below $1 \mathrm{~g} \mathrm{~m}^{-3} 84 \%$ of the time, and below $2 \mathrm{~g} \mathrm{~m}^{-3} 98 \%$ of the time.

In Fig. $5 \mathrm{c}$ we bin the measurements by height to assess the bias dependence in various levels of the atmosphere, and present the resulting binned distributions as boxplots. It is interesting to note that the variance of the bias distribution decreases with height, which at first seems counterintuitive given that measurement SNR is largest in the lowest levels of the atmosphere. However, recall that the strongest biases in the DAR humidity measurement arise from sharp spatial gradients of the characteristic DSD diameter, which are likely to occur in the lower atmosphere (e.g., melting layer, localized precipitation populations). Nevertheless, the near-zero median bias in each vertical level reveals that the temporalaveraging approach successfully mitigates systematic DAR biases. In future DAR measurement systems, it will be interesting to explore the addition of a third transmission frequency outside of the $167-174.8 \mathrm{GHz}$ band to independently measure the frequency-dependent hydrometeor scattering contributions to the differential absorption signal.

\section{b. Surface-to-cloud-base column retrievals}

We utilize all of the cloud datasets that do not feature strong precipitation to perform a measurement of IWV between the radar and cloud base. An example cloud 

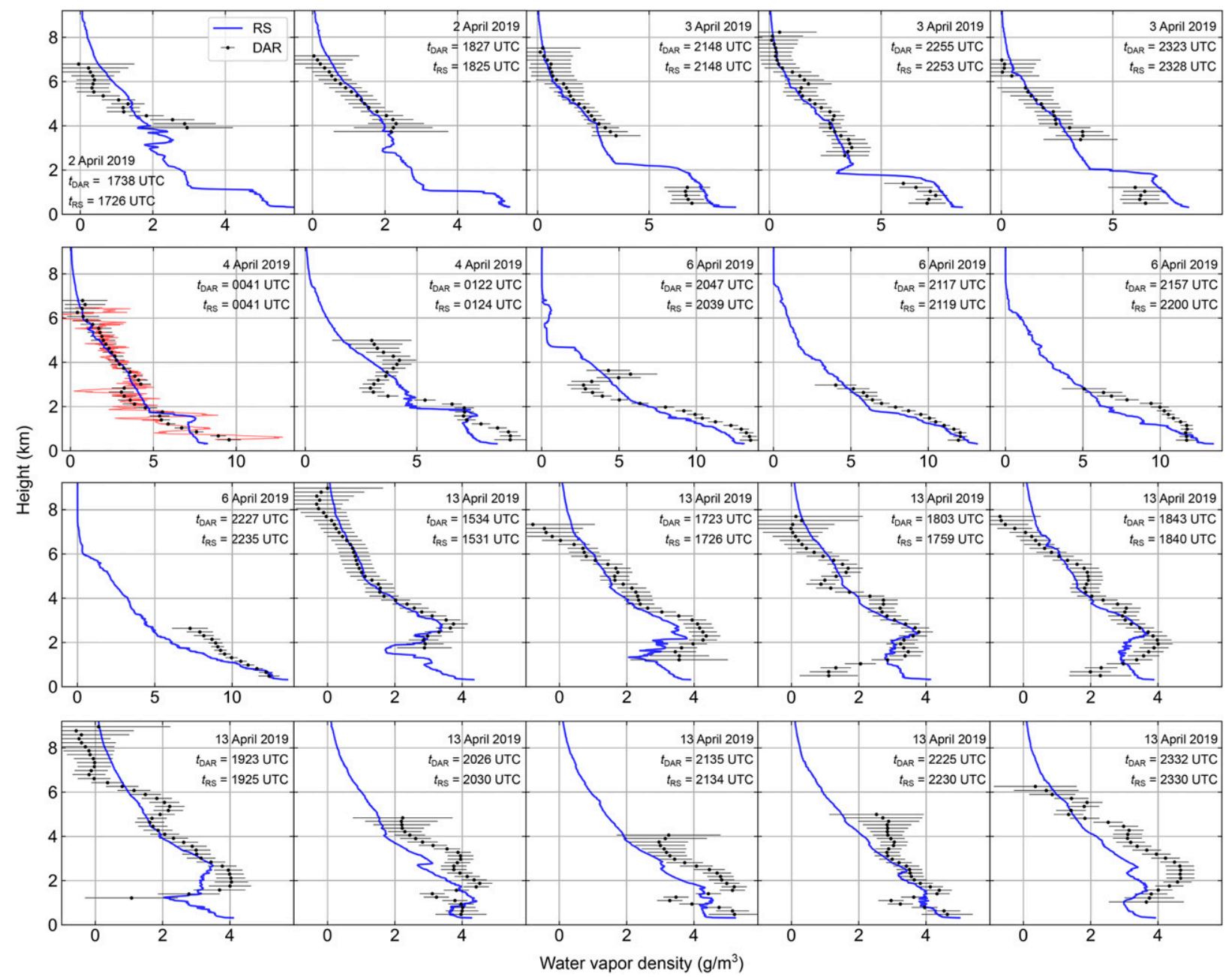

FIG. 4. Comparisons of coincident radiosonde and DAR humidity profiles. The DAR profiles, which are initially retrieved with $5 \mathrm{~s}$ temporal resolution, are further averaged in time to $10 \mathrm{~min}$ resolution for comparison with radiosonde profiles. Error bars for the DAR measurements correspond to the square root of the variance defined in Eq. (21). For comparison, the left panel in the second row also shows the 10-min-averaged humidity profile retrieved using the simple two-frequency DAR method from Roy et al. (2018) (red line).

scene and resulting IWV time series is presented in Fig. 6. In all of the DAR IWV measurements described in this section, we do not perform any additional averaging in time, and so the temporal resolution is $5 \mathrm{~s}$. For the RS time series (blue line in Fig. 6b), we again interpolate the profiles linearly in time between launches at each height, and integrate the resulting profiles up to the cloud-base height determined by radar measurement. For this upper-tropospheric ice-cloud case, the remotely sensed and in situ IWV time series agree very well, even with the assumption of pure Rayleigh backscattering from spherical liquid hydrometeors. We note here that these DAR retrievals utilize the value for the radar calibration ratio $C\left(f_{1}\right) / C\left(f_{2}\right)$ determined from the aggregate analysis described below.

For the case in Fig. 6, a radiosonde was launched at 2330 UTC, making the potential error from linear interpolation in time small. However, many of the datasets used for IWV measurements do not feature the same density of RS launches as those used for in-cloud profiling, since these were the highest-priority measurements. Fortunately, the ARM RL performs measurements of water vapor profiles in clear air every $10 \mathrm{~min}$, and provides a valuable resource for validation of DAR surface-to-cloud-base IWV measurements. However, it is often the case that the RL profiles do not extend all the way to the cloud-base height detected by VIPR. Thus, we adopt an approach that merges the RS and RL water vapor information to interpolate the RSintegrated IWV in time with the RL temporal resolution. Specifically, given an RL humidity profile we first filter out values with uncertainties in excess of $2 \mathrm{~g} \mathrm{~m}^{-3}$, resulting in a maximum RL measurement height $z_{\mathrm{RL}}$. Then, we integrate the RL and temporally interpolated 
(a)

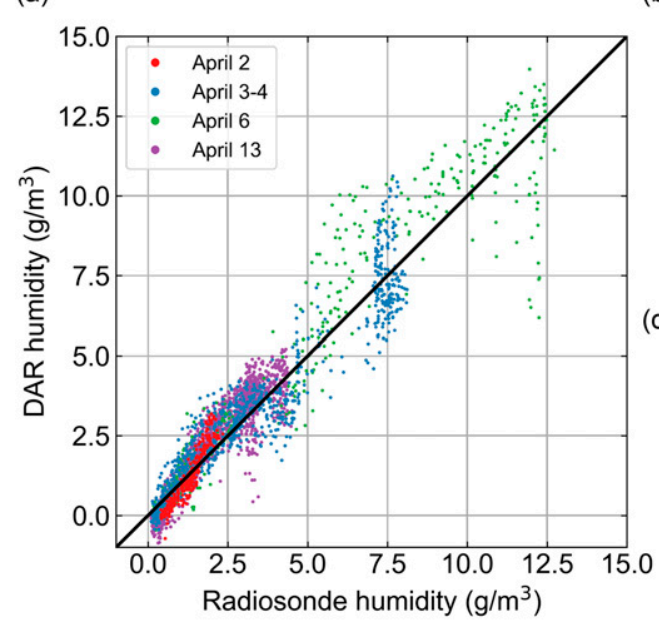

(b)

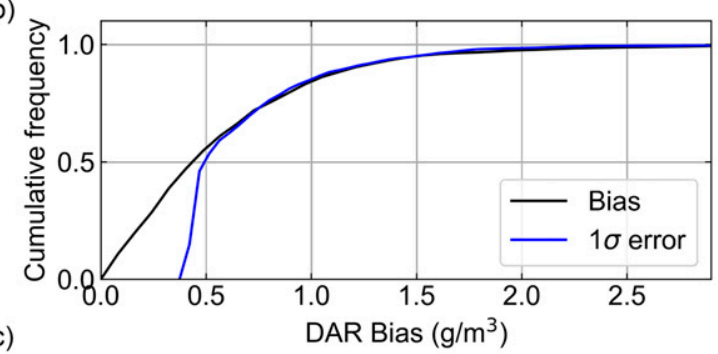

(c)

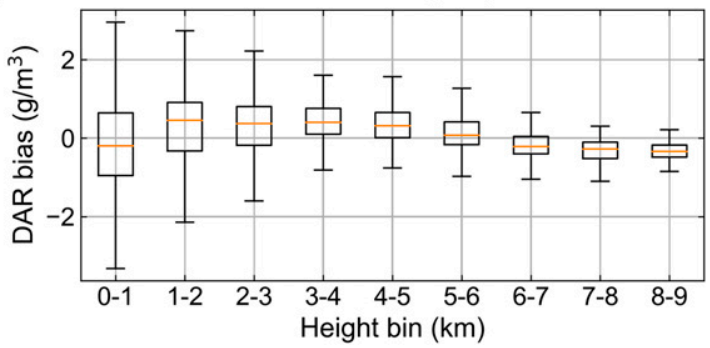

FIG. 5. Summary statistics of in-cloud humidity profiling measurements performed in the ARM deployment. (a) $10 \mathrm{~min}$ averaged DAR humidity profiles ( $180 \mathrm{~m}$ resolution) vs temporally interpolated RS profiles. Measurements from different days are shown in different colors. Fitting a line to the data gives a slope of 1.01 and an intercept of $0.13 \mathrm{~g} \mathrm{~m}^{-3}$, highlighting the close agreement between remotely sensed and in situ measurements. (b) Cumulative probability distribution of absolute value of DAR biases relative to RS profiles (black line) and retrieval precision (blue line) for $10 \mathrm{~min}$ averaged humidity profiles. (c) Tukey box plot of height-binned biases.

RS profiles at each 10 min interval up to $z_{\mathrm{RL}}$, and take the ratio $\mathrm{IWV}_{\mathrm{RL}} / \mathrm{IWV} \mathrm{V}_{\mathrm{RS}}$. This provides a correction factor that can be applied to the temporally interpolated RS profiles for a more accurate comparison with DAR. An example time series of this ratio is shown in Fig. 7a, where we plot both the RL and DAR ratios with respect to the RS IWV. In this case, the nearest RS launches occurred at 1724 and 2039 UTC. The strong agreement between RL and DAR reveal the utility of using this ancillary measurement, as otherwise we would incorrectly determine the DAR IWV to be dry-biased by more than $10 \%$ for much of this measurement window.

Using the RS-RL-merged IWV dataset as ground truth, we aggregate DAR measurements from many days between 2 and 14 April 2019, and perform the modified retrieval algorithm described in section $3 \mathrm{c}$. To determine the value of $C\left(f_{1}\right) / C\left(f_{2}\right)$, we calculate the mean bias for each measurement day before averaging these individual biases together to get a final value. This ensures that datasets with many more time points are not weighted disproportionately, which is desirable because most of the variation in DAR IWV is expected to be due to systematic errors that will depend on the type of cloud measured and its height, as well as radar parameters that could vary on the time scale of days. The chosen value for $10 \log _{10} C\left(f_{1}\right) / C\left(f_{2}\right)=3.6 \mathrm{~dB}$ is the one that yields an average bias of zero, and is close to those measured on three separate days $(4.3,5.1$, and $3.5 \mathrm{~dB})$ with different humidity conditions and two differentsized calibration spheres.

The scatterplot of DAR versus RS-RL IWV values is shown in Fig. 7b, where measurements from different days are depicted with different colors. The large dynamic range of values sampled is a result of measuring

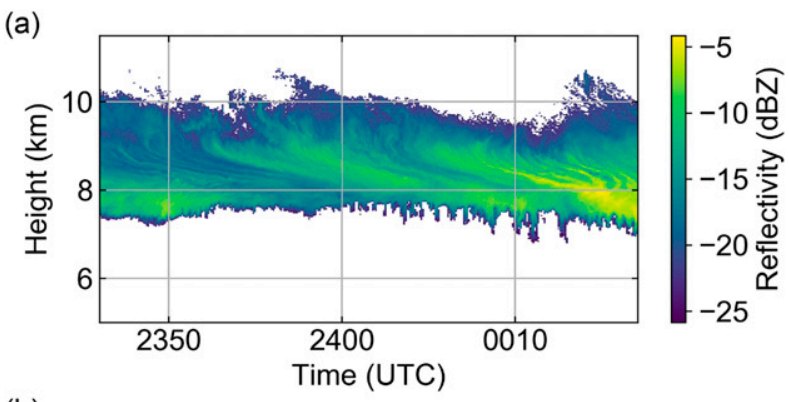

(b)

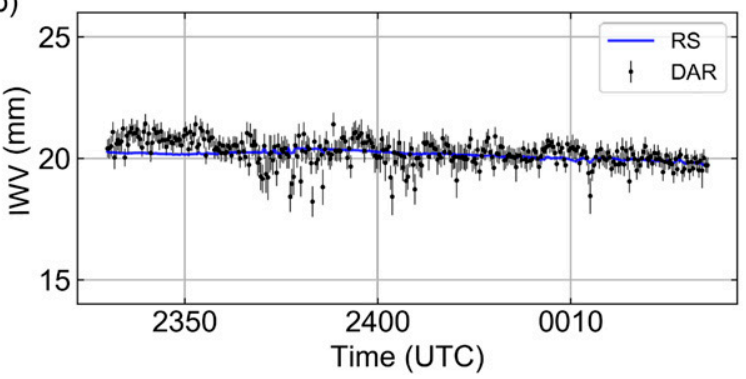

FIG. 6. (a) Observed reflectivity at $f_{1}=167 \mathrm{GHz}$ and (b) corresponding retrieved surface-to-cloud-base IWV with RS-integrated value (blue line) on 5-6 Apr 2019. 

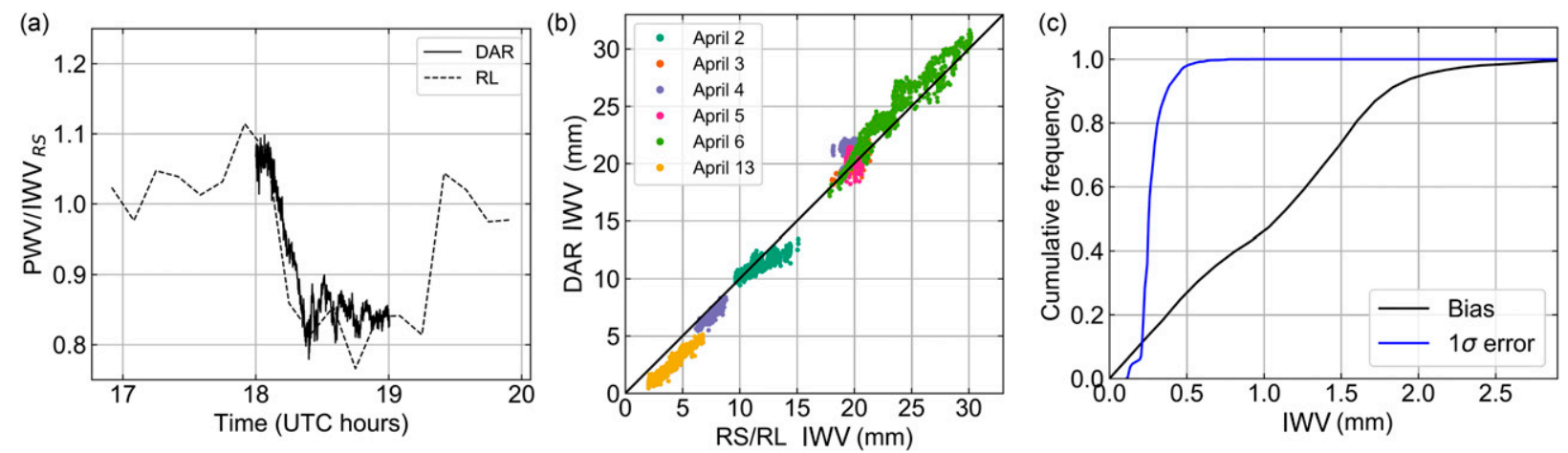

FIG. 7. (a) Time series of the ratio of remotely sensed to RS-integrated IWV for measurements on 6 Apr 2019, where radiosondes were launched at 1724 and 2039 UTC. (b) Comparison of DAR surface-to-cloud-base IWV with RS-RL-merged dataset. The DAR time resolution is $5 \mathrm{~s}$. Measurements from different days are shown in different colors. (c) Cumulative probability distributions for DAR bias (black line) with respect to RS-RL values and $1 \sigma$ retrieval error (blue line).

clouds with different base levels, and of measuring on different days with varying absolute humidities. The correlation coefficient for this comparison is $r=1.00$ with a RMSE of $1.2 \mathrm{~mm}$, and the slope of a best-fit line to the measurements is 1.1. There is a clear trend in the bias in this dataset that is negative for low IWV and positive for high IWV values. We note that as in the case of incloud profiling, the sensitivity of the DAR measurement to IWV is set in absolute terms (i.e., in units of $\mathrm{mm}$ ), and not in terms of relative error. Therefore, there is no reason to expect higher absolute biases for higher IWV values. The resulting cumulative distributions for both the absolute value of measurement bias and the estimated humidity uncertainty from the retrieval ( $1 \sigma$ error) are shown in Fig. $7 \mathrm{c}$. Recall that the uncertainty estimates include contributions from the random error associated with the radar power measurements and systematic error associated with the frequency-dependence of the cloud reflectivity. From Fig. $7 \mathrm{c}$ we find that the median $1 \sigma$ error is $0.25 \mathrm{~mm}$ and the median bias relative to the RS-RL values is $1.1 \mathrm{~mm}$. Furthermore, biases are less than $1 \mathrm{~mm} 46 \%$ of the time, and less than $2 \mathrm{~mm} 95 \%$ of the time.

\section{Conclusions}

The ground-based validation measurements in this work demonstrate for the first time the ability to accurately profile water vapor inside cloudy and precipitating volumes using DAR. Additionally, high-precision measurements of surface-to-cloud-base IWV with RMSE of $1.2 \mathrm{~mm}$ highlight DAR's ability to provide useful measurements in cloud-free regions as well, and are analogous to a total column water vapor measurement between aircraft (or spacecraft) and the ground. If performed from a spaceborne platform, DAR measurements could provide key information on the thermodynamic state of the atmosphere in areas where existing spaceborne technologies cannot probe, including in deep convective regions and regions of strong precipitation within frontal systems. For instance, given a measurement scene with $N_{c}$ cloud layers and provided that hydrometeor extinction is not too severe, a spaceborne DAR could in principle measure 1) IWV between the top of the atmosphere and the top of the highest cloud, 2) continuous humidity profiles inside each of the $N_{c}$ clouds, 3) $N_{c}-1$ values of IWV between the cloud layers, and (4) IWV between the surface and lowest cloud base.

Near-future deployment of VIPR from an airborne platform will offer increased sampling of cloudy atmospheres, because in this case the beam originates from a region of low absolute humidity and therefore low attenuation. By using ground returns from land and ocean surfaces, we will further investigate the ability to retrieve total column water vapor using DAR. Additionally, airborne deployment is necessary for assessing challenges in G-band DAR measurements from a fastmoving platform, including effects from Doppler shifts and the trade-offs between along-track averaging, humidity precision, and profile resolution. Such measurements will help clarify the requirements necessary for a spaceborne DAR instrument.

There are multiple areas in which technological innovation is needed to enhance the sensitivity and accuracy of water vapor DAR. First, reducing the impact of systematic biases from hydrometeor scattering requires the implementation of a third transmission frequency farther from the water line center. Potential locations for this are the nearby $151.5-155.5$ and $158.5-164 \mathrm{GHz}$ bands that feature similar transmission restrictions to that currently utilized in VIPR. Accessing these bands would require substantially increasing the transmitter 
bandwidth. Second, innovation in the area of compact, high-transmit-power G-band sources is critical for realizing a future spaceborne system. Even for ground-based and airborne G-band systems, higherpower transmitters would increase system sensitivity by allowing for finer range resolution and therefore more statistically independent samples per pulse.

Of particular interest for future DAR measurement campaigns would be deployment alongside a water vapor DIAL. This synergistic combination of active humidity profiling technologies makes possible continuous, high-resolution water vapor measurements throughout clear, cloudy, and precipitating regions. This unprecedented sampling of the water vapor distribution could provide key insights into processes with strong coupling to the thermodynamic structure.

Acknowledgments. This research was supported by NASA's Earth Science Technology Office under the Instrument Incubator Program, and was carried out at the Jet Propulsion Laboratory (JPL), California Institute of Technology, Pasadena, California, under contract with the National Aeronautics and Space Administration (NASA). This research was also supported by the Atmospheric Radiation Measurement (ARM) User Facility, a U.S. Department of Energy (DOE) Office of Science user facility managed by the Office of Biological and Environmental Research. Data were obtained from the ARM User Facility, a U.S. DOE Office of Science user facility managed by the Office of Biological and Environmental Research. R. Roy's research was supported by an appointment to the NASA Postdoctoral Program at JPL, administered by Universities Space Research Association under contract with NASA.

Data availability statement: The G-band radar data presented in this work can be made available by the corresponding author upon request.

\section{APPENDIX}

\section{Radar Calibration}

To acquire calibrated measurements of cloud reflectivities, the calibration factor $C(f)$ must be known. By comparing the standard weather radar equation (Doviak and Zrnić 1993) with the reflectivity factor definition $Z_{\text {obs }}=\lambda^{4} \eta_{\text {obs }} /\left[\pi^{5}\left|K_{w}(f)\right|^{2}\right]$, we find an expression for this calibration factor in terms of radar system parameters:

$$
C(f)=\frac{\lambda^{4}}{\pi^{5}\left|K_{w}(f)\right|^{2}} \frac{(4 \pi)^{3}}{P_{t}(f) G_{a}^{2} G_{\mathrm{rec}} \Omega \lambda^{2} \Delta r} .
$$

Here $\eta_{\text {obs }}$ is the observed volume backscattering coefficient, $K_{w}(f)=\left[\varepsilon_{w}(f)-1\right] /\left[\varepsilon_{w}(f)+2\right], \varepsilon_{w}(f)$ is the complex dielectric constant of liquid water, which depends on both frequency and temperature, $P_{t}(f)$ is the radar transmit power, $G_{a}$ is the antenna gain of the quasioptical system including losses, $G_{\text {rec }}$ is the total gain of the receiver chain, $\Omega$ is the two-way solid angle, and $\Delta r$ is the range resolution. For a Gaussian beam power distribution with $1 / e$ angular radius $\theta_{0}$, the two-way solid angle is simply $\Omega=\pi \theta_{0}^{2} / 2$. In keeping with convention, we express all cloud reflectivities as $\mathrm{dB} Z_{\mathrm{obs}}=10 \log _{10}\left(Z_{\mathrm{obs}} / Z_{0}\right)$, where $Z_{0}=1 \mathrm{~mm}^{6} \mathrm{~m}^{-3}$. Furthermore, it is important to note that the reflectivity factor definition necessitates the choice of some nominal value for $\left|K_{w}(f)\right|^{2}$. In this work, we use the frequency-dependent values at $T=$ $280 \mathrm{~K}$ and note that, while $\left|K_{w}(f)\right|^{2}$ varies by roughly $20 \%$ from $0^{\circ}$ to $25^{\circ} \mathrm{C}$, the ratio $\left|K_{w}\left(f_{2}\right)\right|^{2} /\left|K_{w}\left(f_{1}\right)\right|^{2}$ varies by less than $0.2 \%$, implying that the differential absorption measurement is not impacted by this choice.

In theory, all of the parameters in Eq. (A1) can be individually measured or modeled to determine the value of $C(f)$. However, in practice it is more useful to measure the signal strength from a target with wellknown radar cross section placed in the beam path, and thereby calibrate the entire transceiver chain with a single measurement. To this end, we performed calibration measurements using two high-sphericity metal calibration spheres (Century Metal Spinning Co., Model RCS 0200 and 0400) with diameters of $d_{\mathrm{cs}}=2$ and $4 \mathrm{in}$. $(1 \mathrm{in} .=2.54 \mathrm{~cm})$, placed at a range of $r_{\mathrm{cs}}=465 \mathrm{~m}$ and suspended from a 30 foot $(\sim 9 \mathrm{~m})$ pole using thin nylon thread. We can relate the measured echo power from the calibration sphere to unknown quantities in Eq. (A1) using the standard radar equation (Skolnik 2001),

$$
P_{e, \mathrm{cs}}(f)=\frac{P_{t}(f) \lambda^{2} G_{a}^{2} G_{\mathrm{rec}} \sigma_{\mathrm{cs}} e^{-2 \tau\left(r_{\mathrm{cs}} f\right)}}{(4 \pi)^{3} r_{\mathrm{cs}}^{4}},
$$

where $\sigma_{\mathrm{cs}}=\pi d_{\mathrm{cs}}^{2} / 4$ is the radar cross section and the atmospheric attenuation factor requires coincident humidity measurements. In terms of the known calibration sphere measurement variables, the radar calibration factor becomes

$$
C(f)=\frac{\lambda^{4}}{\pi^{5}\left|K_{w}(f)\right|^{2}} \frac{\sigma_{\mathrm{cs}} e^{-2 \tau\left(r_{\mathrm{cs}} f\right)}}{r_{e, \mathrm{cs}}^{4}(f) \Omega \Delta r} .
$$

We performed the radar calibration measurements on three separate days with different temperature and humidity conditions. The alignment of the sphere to the beam center included horizontal adjustments accomplished by varying the anchor point of nylon thread to the ground, and fine zenith angle adjustments using the 
rotation stage on which the radar is mounted, each optimized for maximum radar signal power level. Because absolute calibration at $f_{2}$ is more sensitive to biases stemming from water vapor absorption during the calibration measurement, we only utilize $f_{1}$ for calibrated cloud reflectivity profiles. We estimate our uncertainty in the determination of $C\left(f_{1}\right)$ from the variation over the three measurement days, and find it to be $1 \mathrm{~dB}$. Therefore, all measured reflectivities reported in this work have an associated calibration uncertainty of $1 \mathrm{~dB}$. Note that this uncertainty estimate includes potential misalignment of the calibration sphere and center of the beam, day-to-day drifts in radar transmit power, receiver gain, and uncertainty from not knowing the radar-to-calibration-sphere water vapor column perfectly.

\section{REFERENCES}

Anderson, E., 2018: Statement of guidance for global numerical weather prediction (NWP). World Meteorological Organization, accessed 16 December 2019, https://www.wmo.int/pages/prog/ www/OSY/GOS-RRR.html.

ARM, 2015: Raman lidar (RLPROFMRTEMPCAL), updated hourly. ARM Data Center, accessed 22 May 2019, https:// doi.org/10.5439/1415137.

Battaglia, A., and P. Kollias, 2019: Evaluation of differential absorption radars in the $183 \mathrm{GHz}$ band for profiling water vapour in ice clouds. Atmos. Meas. Tech., 12, 3335-3349, https:// doi.org/10.5194/amt-12-3335-2019.

— C. D. Westbrook, S. Kneifel, P. Kollias, N. Humpage, U. Löhnert, J. Tyynelä, and G. W. Petty, 2014: G band atmospheric radars: New frontiers in cloud physics. Atmos. Meas. Tech., 7, 1527-1546, https://doi.org/10.5194/amt-7-1527-2014.

Blumberg, W. G., D. D. Turner, U. Löhnert, and S. Castleberry, 2015: Ground-based temperature and humidity profiling using spectral infrared and microwave observations. Part II: Actual retrieval performance in clear-sky and cloudy conditions. J. Appl. Meteor. Climatol., 54, 2305-2319, https://doi.org/ 10.1175/JAMC-D-15-0005.1.

Bohren, C., and D. Huffman, 2004: Absorption and Scattering of Light by Small Particles. Wiley, 544 pp., https://doi.org/ 10.1002/9783527618156.

Browell, E. V., T. D. Wilkerson, and T. J. Mcilrath, 1979: Water vapor differential absorption lidar development and evaluation. Appl. Opt., 18, 3474-3483, https://doi.org/10.1364/ AO.18.003474.

—, S. Ismail, and W. Grant, 1998: Differential absorption lidar (DIAL) measurements from air and space. Appl. Phys., 67B, 399-410, https://doi.org/10.1007/s003400050523.

Cooper, K. B., R. R. Monje, L. Millán, M. Lebsock, S. Tanelli, J. V. Siles, C. Lee, and A. Brown, 2018: Atmospheric humidity sounding using differential absorption radar near $183 \mathrm{GHz}$. IEEE Geosci. Remote Sens. Lett., 15, 163-167, https://doi.org/ 10.1109/LGRS.2017.2776078.

—_, and Coauthors, 2020: A G-band radar for humidity and cloud remote sensing. IEEE Trans. Geosci. Remote Sens., https://doi.org/10.1109/TGRS.2020.2995325, in press

Doviak, R., and D. Zrnić, 1993: Doppler Radar and Weather Observations. 2nd ed. Dover Publications, 562 pp.
Lebsock, M. D., K. Suzuki, L. F. Millán, and P. M. Kalmus, 2015: The feasibility of water vapor sounding of the cloudy boundary layer using a differential absorption radar technique. Atmos. Meas. Tech., 8, 3631-3645, https://doi.org/10.5194/amt8-3631-2015.

Lipton, A. E., 2003: Satellite sounding channel optimization in the microwave spectrum. IEEE Trans. Geosci. Remote Sens., 41, 761-781, https://doi.org/10.1109/TGRS.2003.810926.

Maddy, E. S., and C. D. Barnet, 2008: Vertical resolution estimates in version 5 of AIRS operational retrievals. IEEE Trans. Geosci. Remote Sens., 46, 2375-2384, https://doi.org/10.1109/ TGRS.2008.917498.

Meneghini, R., L. Liao, and L. Tian, 2005: A feasibility study for simultaneous estimates of water vapor and precipitation parameters using a three-frequency radar. J. Appl. Meteor., 44, 1511-1525, https://doi.org/10.1175/JAM2302.1.

Millán, L., M. Lebsock, N. Livesey, and S. Tanelli, 2016: Differential absorption radar techniques: Water vapor retrievals. Atmos. Meas. Tech., 9, 2633-2646, https://doi.org/10.5194/amt-9-26332016.

NASEM, 2018: Thriving on Our Changing Planet: A Decadal Strategy for Earth Observation from Space. National Academies Press, 716 pp., https://doi.org/10.17226/24938.

Nehrir, A. R., K. S. Repasky, and J. L. Carlsten, 2011: Eye-safe diode-laser-based micropulse differential absorption lidar (DIAL) for water vapor profiling in the lower troposphere. J. Atmos. Oceanic Technol., 28, 131-147, https://doi.org/ 10.1175/2010JTECHA1452.1.

__ and Coauthors, 2017: Emerging technologies and synergies for airborne and space-based measurements of water vapor profiles. Surv. Geophys., 38, 1445-1482, https://doi.org/10.1007/ s10712-017-9448-9.

NTIA, 2015: Manual of regulations and procedures for federal radio frequency management. NTIA Doc., 829 pp., https:// www.ntia.doc.gov/page/2011/manual-regulations-and-proceduresfederal-radio-frequency-management-redbook.

Read, W. G., Z. Shippony, and W. Snyder, 2004: Earth Observing System (EOS) Microwave Limb Sounder (MLS) forward model algorithm theoretical basis document. Jet Propulsion Laboratory Tech. Rep. JPL D-18130/CL\#04-2238, 248 pp.

Rodgers, C., 2000: Inverse Methods for Atmospheric Sounding: Theory and Practice. Series on Atmospheric, Oceanic and Planetary Physics, Vol. 2, World Scientific, 238 pp., https:// doi.org/10.1142/3171.

Roy, R. J., M. Lebsock, L. Millán, R. Dengler, R. Rodriguez Monje, J. V. Siles, and K. B. Cooper, 2018: Boundary-layer water vapor profiling using differential absorption radar. Atmos. Meas. Tech., 11, 6511-6523, https://doi.org/10.5194/ amt-11-6511-2018.

Sahoo, S., X. Bosch-Lluis, S. C. Reising, and J. Vivekanandan, 2015: Radiometric information content for water vapor and temperature profiling in clear skies between 10 and $200 \mathrm{GHz}$. IEEE J. Sel. Top. Appl. Earth Obs. Remote Sens., 8, 859-871, https://doi.org/10.1109/JSTARS.2014.2364394.

Santanello, J. A., and Coauthors, 2018: Land-atmosphere interactions: The LoCo perspective. Bull. Amer. Meteor. Soc., 99, 1253-1272, https://doi.org/10.1175/BAMS-D-17-0001.1.

Siles, J. V., K. B. Cooper, C. Lee, R. H. Lin, G. Chattopadhyay, and I. Mehdi, 2018: A new generation of room-temperature frequency-multiplied sources with up to $10 \mathrm{x}$ higher output power in the $160 \mathrm{GHz}-1.6 \mathrm{THz}$ range. IEEE Trans. Terahertz Sci. Technol., 8, 596-604, https://doi.org/10.1109/ TTHZ.2018.2876620. 
Skolnik, M., 2001: Introduction to Radar Systems. 3rd ed. McGrawHill, 772 pp.

Spuler, S. M., K. S. Repasky, B. Morley, D. Moen, M. Hayman, and A. R. Nehrir, 2015: Field-deployable diode-laser-based differential absorption lidar (DIAL) for profiling water vapor. Atmos. Meas. Tech., 8, 1073-1087, https://doi.org/10.5194/amt8-1073-2015.

Tian, B., E. J. Fetzer, and E. M. Manning, 2019: The atmospheric infrared sounder Obs4MIPs version 2 data set. Earth Space Sci., 6, 324-333, https://doi.org/10.1029/ 2018EA000508.

Turner, D. D., and J. E. M. Goldsmith, 1999: Twenty-four-hour Raman lidar water vapor measurements during the Atmospheric Radiation Measurement program's 1996 and 1997 water vapor intensive observation periods. J. Atmos. Oceanic Technol., 16, 1062-1076, https://doi.org/10.1175/1520-0426(1999)016<1062: TFHRLW $>2.0 . \mathrm{CO} ; 2$.

- and U. Löhnert, 2014: Information content and uncertainties in thermodynamic profiles and liquid cloud properties retrieved from the ground-based Atmospheric Emitted Radiance Interferometer (AERI). J. Appl. Meteor. Climatol., 53, 752771, https://doi.org/10.1175/JAMC-D-13-0126.1.
_ R. A. Ferrare, and L. A. Brasseur, 2001: Average aerosol extinction and water vapor profiles over the Southern Great Plains. Geophys. Res. Lett., 28, 4441-4444, https://doi.org/ 10.1029/2001GL013691.

_ J. E. M. Goldsmith, and R. A. Ferrare, 2016: Development and applications of the ARM Raman lidar. The Atmospheric Radiation Measurement Program: The First 20 Years, Meteor. Monogr., No. 57, Amer. Meteor. Soc., https://doi.org/10.1175/ AMSMONOGRAPHS-D-15-0026.1.

Weckwerth, T. M., K. J. Weber, D. D. Turner, and S. M. Spuler, 2016: Validation of a water vapor micropulse differential absorption lidar (DIAL). J. Atmos. Oceanic Technol., 33, 2353 2372, https://doi.org/10.1175/JTECH-D-16-0119.1.

Whiteman, D. N., S. H. Melfi, and R. A. Ferrare, 1992: Raman lidar system for the measurement of water vapor and aerosols in the Earth's atmosphere. Appl. Opt., 31, 3068-3082, https://doi.org/ 10.1364/AO.31.003068.

Wulfmeyer, V., and Coauthors, 2015: A review of the remote sensing of lower tropospheric thermodynamic profiles and its indispensable role for the understanding and the simulation of water and energy cycles. Rev. Geophys., 53, 819-895, https:// doi.org/10.1002/2014RG000476. 